\title{
Dynamical Analysis and Design of Active Orthoses for Spinal Cord Injured Subjects by Aesthetic and Energetic Optimization
}

\author{
García-Vallejo, D. ${ }^{1 *}$, Font-Llagunes, J.M. ${ }^{2}$, Schiehlen, W. ${ }^{3}$ \\ ${ }^{1}$ Department of Mechanical Engineering and Manufacturing, University of Seville, \\ Camino de los Descubrimientos s/n, 41092 Seville, Spain. \\ ${ }^{2}$ Biomechanical Engineering Group, Department of Mechanical Engineering and \\ Biomedical Engineering Research Centre, Universitat Politècnica de Catalunya, \\ Diagonal 647, 08028 Barcelona, Spain. \\ ${ }^{3}$ Institute of Engineering and Computational Mechanics, University of Stuttgart, \\ Pfaffenwaldring 9, 70569 Stuttgart, Germany.
}

\begin{abstract}
The dynamic analysis and simulation of human gait using multibody dynamics techniques has been a major area of research in the last decades. Nevertheless, not much attention has been paid to the analysis and simulation of robotic-assisted gait. Simulation is a very powerful tool both for assisting the design stage of active rehabilitation robots, and predicting the subject-orthoses cooperation and the resulting aesthetic gait. This paper presents a parameter optimization approach that allows simulating gait motion patterns in the particular case of a subject with incomplete spinal cord injury (SCI) wearing active knee-ankle-foot orthoses at both legs. The subject is modelled as a planar multibody system actuated through the main lower limb muscle groups. A muscle force-sharing problem is solved to obtain optimal muscle activation patterns. Furthermore, denervation of muscle groups caused by the SCI is parameterized to account for different injury severities. The active orthoses are modelled as external devices attached to the legs, and their dynamic and performance parameters are taken from a real prototype. Numerical results using energetic and aesthetic objective functions, and considering different SCI severities are obtained. Detailed discussions are given related to the different motion and actuation patterns both from muscles and orthoses. The proposed methodology opens new perspectives towards the prediction of human-assisted gait, which can be very helpful for the design of new rehabilitation robots.
\end{abstract}

Keywords: Human gait, active orthosis, parameter optimization, spinal cord injury, energetics, aesthetics.

\section{Introduction}

Gait analysis by computational mechanics techniques has been a major area of research interest for many years. Multibody system dynamics (MSD) techniques are potentially very powerful in this field and there are many contributions from the MSD community to this challenging problem $[1,2,3,4]$. Among other approaches, parameter optimization techniques have been frequently used for motion synthesis of biped robots [5]. These optimization techniques have been proven to

\footnotetext{
*Corresponding Author. e-mail: dgvallejo@us.es
} 
be also a powerful tool in human walking dynamics research $[6,7]$. In these works, muscle forces and generalized coordinates are described in terms of a certain set of parameters, whose optimal values are found by minimizing cost functions that include an energy expenditure estimation and a measure of deviation from normal gait patterns. This method is mainly based on inverse dynamics since at each iteration of the optimization algorithm an inverse dynamics problem is solved by using the motion reconstructed from the design parameters. The main advantage of this approach is the complete elimination of the forward time integrations of the equations of motion, which significantly reduces the computational cost of simulation.

In order to study human gait dynamics, planar models have the advantage of being efficient and accurate enough to analyze symmetrical walking patterns. As an example, Ackermann [6] used a two-dimensional (2D) model to analyze the walking motion of humans with bilateral disorders, which are in fact less common than unilateral disorders. Based on this approach, García-Vallejo and Schiehlen [7] developed a three-dimensional (3D) model to simulate unilateral disorders. Most of these models are composed of seven bodies ( 2 feet, 2 shanks, 2 thighs and a pelvis-trunk body) or eight bodies (2 feet, 2 shanks, 2 thighs, pelvis and separate trunk), where the arms and head are lumped into the trunk body by adding its mechanical properties to this body and ignoring their own dynamics. Umberger [8] has studied the influence of the arms swing motion on the kinematics, kinetics and energetics of human gait reporting an influence less than $10 \%$. The high interest in human walking dynamics has favored the appearance of specific software for model development. It is worth mentioning the work by Delp et al. [9], who developed a graphic-based software system for creating and analyzing spatial dynamic simulations of human movement.

Although several studies have been performed in the last decades related to human walking dynamics, not much attention has been paid to the analysis and simulation of human gait assisted by active orthoses or exoskeletons. In fact, most of the current robotic (or active) orthoses are designed and built without taking into account the coupled dynamic behaviour of the humanorthosis system. It has been shown that robotic actuation is useful for neurorehabilitation and lower limb motor function recovery [10]. Thus, a number of robotic orthoses and exoskeletons aimed at assisting human gait have been developed in research laboratories [11]. For example, Blaya and Herr [12] developed an active ankle-foot orthosis (AFO) to assist drop-foot gait, in which a linear series elastic actuator (SEA) is used to assist ankle motion. Plantar sensors and potentiometers are recruited to identify gait phases. Knee-ankle-foot orthoses (KAFO) are used in patients with more severe gait dysfunctions, including partial or complete paralysis of the lower limbs. One particular type of KAFO is the stance-control KAFO or SCKAFO, respectively, which is well suited for patients with incomplete spinal cord injury (SCI) that can control hip muscles [13]. This device permits free knee motion during swing and locks the knee flexion during stance phase. Font-Llagunes et al. [14] developed a robotic SCKAFO with two parallel systems actuating the knee joint: a controllable shape-locking mechanism "Neuro Tronic" and an electrical actuation system (DC motor plus a planetary gearbox) to assist knee flexion-extension during swing. The prototype is equipped with plantar pressure sensors and joint encoders. Pneumatic artificial muscles (McKibben muscles) are also used in orthotics. A KAFO including 6 artificial muscles, potentiometers, plantar pressure sensors, and electromyography (EMG) is presented in [15]. In that KAFO, the artificial muscles are designed to mimic the agonist-antagonist pairs of the human body. More severe dysfunctions require the use of complete lower limb exoskeletons, like Ekso (Ekso Bionics, USA) [16] or ReWalk (Argo Medical Technologies Inc., Israel) [17] among others.

The aim of this paper is to simulate the orthosis-assisted gait of a subject with incomplete SCI using parameter optimization. Spinal cord injuries cause paralysis of the lower limbs as they break the connections from the central nervous system to the muscular units of the lower body. In the present paper, a planar symmetrical model is used since the assistive devices under consideration are fully symmetric, being designed for SCI subjects affected similarly in both sides 
of the body. Therefore, we consider that the subject wears an identically powered active orthosis on each leg. The subject is modelled as a planar multibody system actuated through the main lower limb muscle groups. So, a muscle force-sharing problem is solved to obtain optimal muscle activation patterns. Denervation of muscle groups caused by the SCI is parameterized to account for different severities of the SCI. The active orthoses are modelled as external devices attached to the legs, and their dynamic and performance parameters are taken from a real prototype presented in [14]. We believe that the presented dynamic simulation methodology could be of great help to computationally predict the subject-orthoses cooperation and resulting gait, and thus, to assist the design of patient-tailored neurorehabilitation devices.

The paper is structured as follows. Section 2 is devoted to describe the design and operation of the considered active orthosis. Next, in Section 3 the human-orthosis multibody model used in the simulations is developed. This section includes the description of the muscle modelling and the inversion of activation and contraction dynamics. Section 4 is aimed at describing the parameter optimization with emphasis on the cost function definition and the constraint formulation. Section 5 presents the obtained numerical results in different simulation cases. Finally, Section 6 contains the final discussion and conclusions of the work.

\section{Description of the Active Orthosis and its Operation}

The design of the orthosis is based on the idea of improving the commercial passive orthoses that SCI patients are using at present. There are different SCI levels according to the standard neurological classification of the American Spinal Injury Association (ASIA). Those are classified by the ASIA Impairment Scale (AIS) and range from A (complete SCI) to E (normal motor and sensory function). The active orthosis that is considered in this paper is aimed at assisting incomplete SCI subjects with AIS level C or D [14]. These levels represent incomplete spinal cord injuries. The target patients preserve motor function of the hip muscles, but have partially denervated muscles controlling the knee and ankle joints. These patients can perform a low-speed, high-cost pathological gait by using walking aids such as crutches, canes or parallel bars.

The current commercial orthoses for the targeted patients include a knee locking system, which is essential to bear the patient's weight during the stance phase due to the lack of force at the quadriceps muscle; and a passive ankle joint (Klenzak joint) that constrains ankle plantar flexion during the swing phase, thus avoiding drop-foot gait. Commercial knee-locking systems are shape or friction-based, being activated upon heel strike detection, usually by means of an on-off contact sensor. The Klenzak joint consists of a spring that applies an external dorsiflexion torque. Those devices are essentially passive, being the only semi-active system that formed by the knee locking mechanism and the on-off contact sensor.

The considered active orthosis, depicted in Fig. 1, includes the following modifications with respect to the current passive devices: a) actuation at the knee joint is added because the considered subjects do not have enough muscle force to flex and extend the lower limb during the swing phase; b) additional sensors are included to better control the knee-locking system and actuation [14]; and c) the standard Klenzak joint is slightly modified including an optical incremental encoder for control purposes. The knee joint incorporates two powered systems acting in parallel: a locking system which locks the knee during stance phase, and an actuation system which is active during swing. The objective of using the two systems is to avoid the use of the motor for locking the knee during stance, thus reducing the power consumption of the orthosis. The first prototype of active orthosis has been tested in a lab environment with SCI subjects as depicted in Fig. 1(b). Moreover, inverse dynamic analyses on healthy subjects have been reported in Lugrís et al. [18].

The operation of the orthosis during the gait cycle is as follows: at initial stance, the contact sensor detects the heel strike and then the knee joint is locked; during this phase the motor does not exert any torque on the joint. During the stance phase the plantar sensors and ankle encoder 


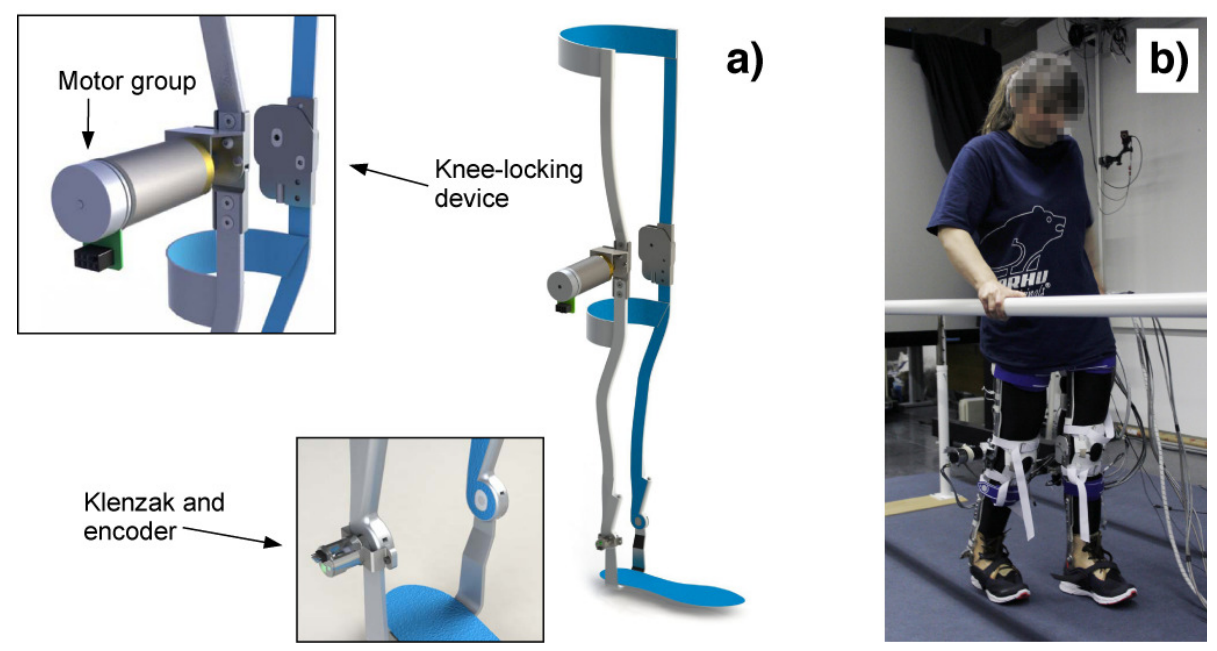

Figure 1: (a) CAD design of the SCKAFO prototype. (b) Experimental test of a SCI subject wearing the two active orthoses.

data give information on the evolution of the gait cycle. Once contact is over (because the other leg has landed on the ground), the locking mechanism is unlocked. Then, the swing phase begins and the knee actuator assists the knee flexion and then extension. The motor control during this phase will be done based on the motor and ankle encoders. After the swing phase the leg makes contact again with the ground and the new cycle begins.

\section{Model Description}

The musculoskeletal model used in this paper is a 2D rigid multibody system actuated by muscles and electrical motors. The equations of motion of the system were obtained by using the multibody software Neweul- $\mathrm{M}^{2}$ [19], which generates the equations of motion in symbolic form for efficiently analyzing, simulating and optimizing multibody systems. The skeleton is first considered as an open kinematic chain built from 7 rigid bodies (two thighs, two shanks, two feet, and a body called HAT representing the pelvis, trunk, arms and head) that are connected by holonomic joints and described by a set of $n_{c}$ generalized coordinates, see Figure 2.

The kinematic chain in Figure 2 is described by the following vector of 9 generalized coordinates

$$
\boldsymbol{y}=\left[\begin{array}{lllllllll}
x_{I 1} & z_{I 1} & \beta_{I 1} & \beta_{13} & \beta_{34} & \beta_{45} & \beta_{16} & \beta_{67} & \beta_{78}
\end{array}\right]^{T}
$$

where the subscript $I$ refers to the inertial frame, subscript 1 refers to body HAT, subscripts 3 and 6 refer to right and left thighs, respectively, subscripts 4 and 7 refer to right and left shanks, respectively, and subscripts 5 and 8 refer to right and left feet, respectively. When a subscript is written as $i j$ it means a relative motion of body $j$ with respect to body $i$.

Based on the Newton-Euler equations of the rigid bodies in the kinematic chain, the equations of motion are written in terms of the generalized coordinates by virtue of d'Alembert's principle $[20]$ as

$$
\boldsymbol{M}(\boldsymbol{y}) \ddot{\boldsymbol{y}}+\boldsymbol{k}(\boldsymbol{y}, \dot{\boldsymbol{y}})=\boldsymbol{q}_{a}(\boldsymbol{y}, \dot{\boldsymbol{y}})+\boldsymbol{B} \boldsymbol{A} \boldsymbol{f}^{\boldsymbol{m}}+\boldsymbol{q}_{a c t}+\boldsymbol{q}_{a n k}(\boldsymbol{y})
$$

where $\boldsymbol{M}(\boldsymbol{y})$ is the $\left(n_{c} \times n_{c}\right)$ - mass matrix of the system, $\boldsymbol{y}, \dot{\boldsymbol{y}}$ and $\ddot{\boldsymbol{y}}$ are the $\left(n_{c} \times 1\right)$ - position, velocity and acceleration vectors, respectively, $\boldsymbol{k}$ is a $\left(n_{c} \times 1\right)$ - vector describing the generalized Coriolis and centrifugal forces, $\boldsymbol{q}_{a}$ is a $\left(n_{c} \times 1\right)$ - vector of applied forces including generalized gravitational forces, passive generalized moments at the joints due to tissues interacting with the joints according to the model of Riener and Edrich [21] and generalized viscous damping torques at the knees and hips according to the model of Stein et al. [22]. $\boldsymbol{B} \boldsymbol{A} \boldsymbol{f}^{\boldsymbol{m}}$ is a $\left(n_{c} \times 1\right)$ - vector 


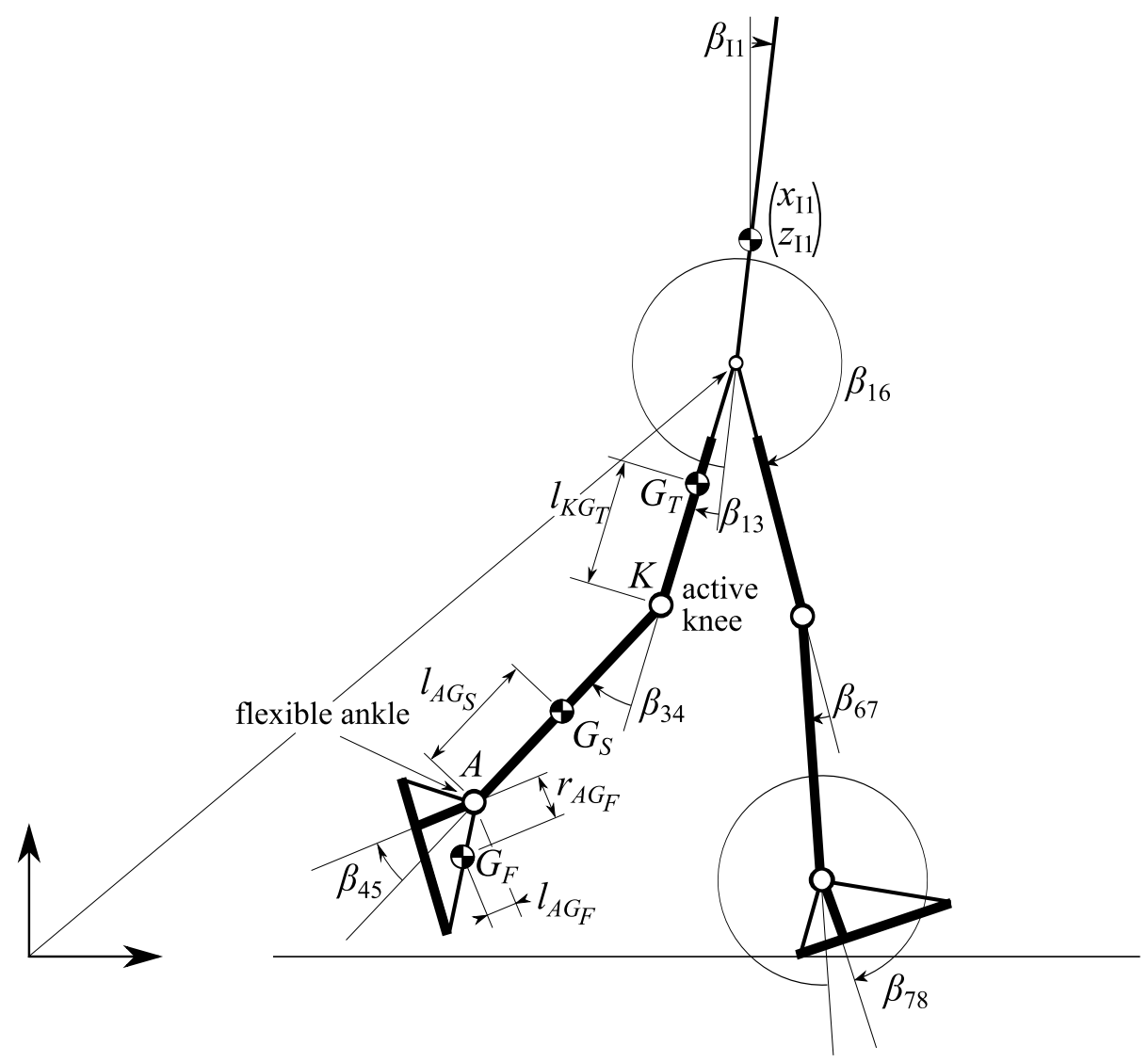

Figure 2: 2D-Model of a subject showing the active SCKAFO in its legs.

that includes the generalized forces exerted by the muscles actuating the model. The $\left(N_{m} \times 1\right)$ vector $f^{m}$ summarizes the forces generated by a reduced set of $N_{m}$ muscles included in the model as described in Appendix A. Matrix $\boldsymbol{A}$ is the constant $\left(n_{b} \times N_{m}\right)$ - matrix of moment arms and is used to calculate the torques generated by all muscles at the actuated joints, where $n_{b}$ is the number of actuated joints, and matrix $\boldsymbol{B}$ is a $\left(n_{c} \times n_{b}\right)$ - distribution matrix used to obtain the generalized torques due to muscle torques at the actuated joints. In Equation (2) $\boldsymbol{q}_{\text {act }}$ and $\boldsymbol{q}_{\text {ank }}(\boldsymbol{y})$ are two $\left(n_{c} \times 1\right)$ - vectors included in the model to account for the orthosis actuation and for the stiffness of the passive Klenzak ankle joint. These vectors are written as follows:

$$
\begin{aligned}
& \boldsymbol{q}_{a c t}=\left[\begin{array}{lllllllll}
0 & 0 & 0 & 0 & T_{k r} & 0 & 0 & T_{k l} & 0
\end{array}\right]^{T} \\
& \boldsymbol{q}_{a n k}(\boldsymbol{y})=\left[\begin{array}{lllllllll}
0 & 0 & 0 & 0 & 0 & T_{a r}(\boldsymbol{y}) & 0 & 0 & T_{a l}(\boldsymbol{y})
\end{array}\right]^{T}
\end{aligned}
$$

where $T_{k r}$ and $T_{k l}$ are the motor torques exerted at the right and left knees and $T_{a r}(\boldsymbol{y})$ and $T_{a l}(\boldsymbol{y})$ are the right and left ankle torques exerted by the flexible ankle joint. $T_{a r}(\boldsymbol{y})$ and $T_{a l}(\boldsymbol{y})$ are evaluated as follows:

$$
\begin{aligned}
& T_{a r}(\boldsymbol{y})=T_{a r}^{0}-k_{a r} \beta_{45} \\
& T_{a l}(\boldsymbol{y})=T_{a l}^{0}-k_{a l} \beta_{78}
\end{aligned}
$$

where $T_{a r}^{0}$ and $T_{a l}^{0}$ are the torques exerted by the passive ankle joint in neutral position $\left(\beta_{45}=0\right.$ and $\left.\beta_{78}=0\right)$, and $k_{a r}$ and $k_{a l}$ are the stiffness coefficients of the passive ankle joints of the orthosis.

The physical parameters of the human body are taken in this work from the work of Ackermann [6]. On the other hand, the physical parameters of the active orthosis are selected to have an orthosis that represents the one designed by Font-Llagunes et al. [14]. These parameters are given in Table 1 with the lengths defined in Fig. 2. 


\begin{tabular}{clc}
\hline \hline$m_{T}$ & Mass of the thigh bars & $0.20 \mathrm{~kg}$ \\
$I_{G_{T}}$ & Inertia moment of the thigh bars about $G_{T}$ & $1.53 \cdot 10^{-3} \mathrm{kgm}^{2}$ \\
$l_{K G_{T}}$ & Distance from $K$ to $G_{T}$ & $0.13 \mathrm{~m}$ \\
\hline$m_{K}$ & Mass of the motor, locking system and knee joint (point mass) & $1.11 \mathrm{~kg}$ \\
\hline$m_{S}$ & Mass of the shank bars & $0.32 \mathrm{~kg}$ \\
$I_{G_{S}}$ & Inertia moment of the shank bars about $G_{S}$ & $4.76 \cdot 10^{-3} \mathrm{kgm}^{2}$ \\
$l_{A G_{S}}$ & Distance from $A$ to $G_{S}$ & $0.17 \mathrm{~m}$ \\
\hline$m_{A}$ & Mass of the encoder at the ankle (point mass) & $0.06 \mathrm{~kg}$ \\
\hline$m_{F}$ & Mass of the foot support & $0.10 \mathrm{~kg}$ \\
$I_{G_{F}}$ & Inertia moment of the foot support about $G_{F}$ & $1.12 \cdot 10^{-4} \mathrm{kgm}^{2}$ \\
$l_{A G_{F}}$ & Distance from $A$ to $G_{F}$ (vertical) & $0.07 \mathrm{~m}$ \\
$r_{A G_{F}}$ & Distance from $A$ to $G_{F}$ (horizontal) & $0.04 \mathrm{~m}$ \\
\hline \hline
\end{tabular}

Table 1: Dynamic parameters of the SCKAFO.

Once the kinematic chain representing the skeleton is described, the contact of the chain with the ground is added. The contact conditions in the different walking phases are represented by unilateral constraints. However, due to the use of an optimization framework in which it is possible to constrain the normal contact forces to be only positive, the contact with the ground is modelled using simple bilateral constraints associated to the joints attached to the feet. Therefore, the contact forces can be easily added to the model by using a vector of Lagrange multipliers as

$$
\boldsymbol{M}(\boldsymbol{y}) \ddot{\boldsymbol{y}}+\boldsymbol{k}(\boldsymbol{y}, \dot{\boldsymbol{y}})=\boldsymbol{q}_{a}(\boldsymbol{y}, \dot{\boldsymbol{y}})+\boldsymbol{B} \boldsymbol{A} \boldsymbol{f}^{\boldsymbol{m}}+\boldsymbol{q}_{a c t}+\boldsymbol{q}_{a n k}(\boldsymbol{y})+\boldsymbol{C}_{p h}^{T} \boldsymbol{\lambda}_{p h} \quad(p h=1,2, \ldots 8)
$$

where $\boldsymbol{C}_{p h}$ is the Jacobian of the active kinematic constraints and $\boldsymbol{\lambda}_{p h}$ is the vector of Lagrange multipliers at phase $p h$ of the motion. Note that the previous equation is used together with constraint equations forcing the normal contact forces to be always positive. Thus, hard impacts will be avoided.

The contact conditions of different phases of the walking cycle are summarized in Figure 3 in agreement with the model of the foot adopted. Note that $H_{r}$ and $T_{r}$ are used to refer to the right heel and right toe, respectively; while $H_{l}$ and $T_{l}$ are used to refer to the left heel and left toe, respectively.
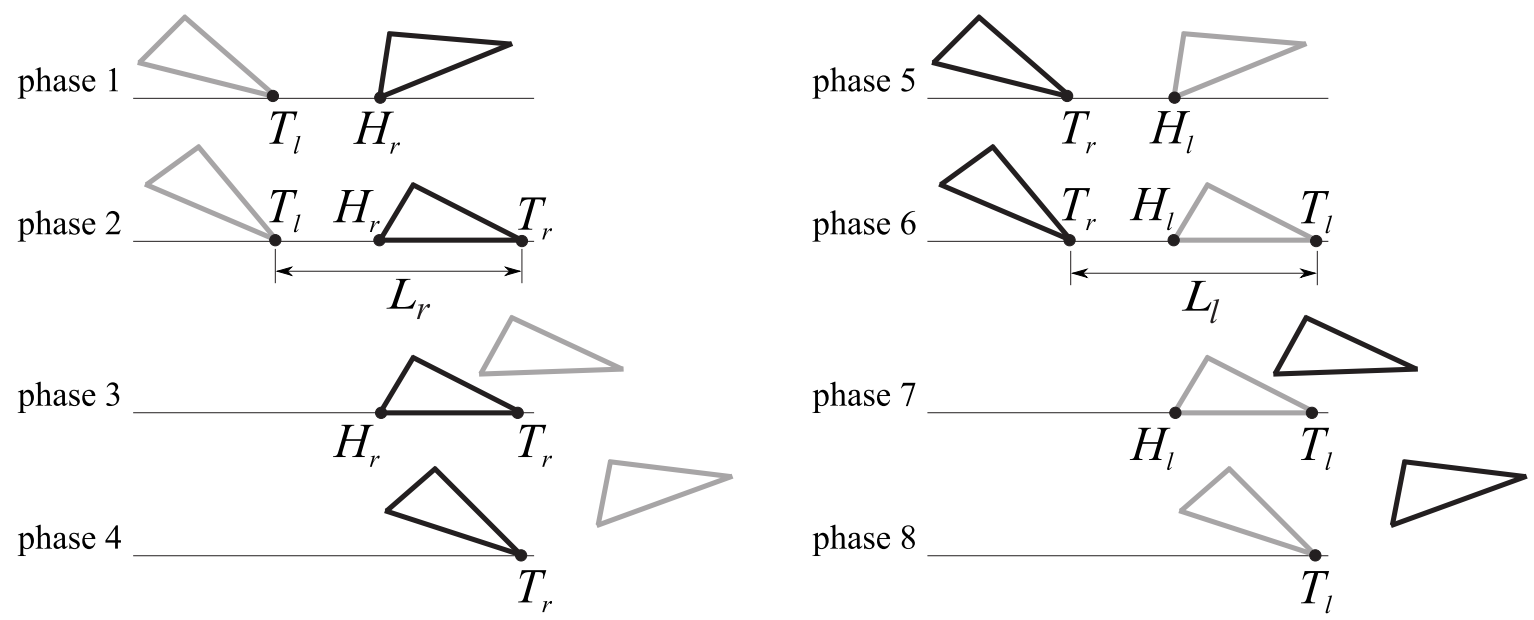

Figure 3: Sketch of the contact conditions for the eight phases of the gait motion.

In the formulation of contact, it is assumed that there is no sliding of the feet during the whole cycle of walking. The contact conditions at the different phases are modelled as follows: 
in phase 1 the left toe contact is modelled by constraining the two displacements of point $T_{l}$ while the right heel contact is modelled by constraining the two displacements of point $H_{r}$; in phase 2 a constraint to the vertical displacement of point $T_{r}$ is added to the constraint set of phase 1 due to the contact of the right toe; in phase 3 the contact at $T_{l}$ is removed; in phase 4 the contact at $H_{r}$ is removed while the right toe contact is modelled by constraining the two displacements of point $T_{r}$; in phase 5 the contact at the left heel is added by constraining the two displacements of point $H_{l}$; in phase 6 a constraint to the vertical displacement of point $T_{l}$ is added to the constraint set of phase 5 due to the contact of the left toe; in phase 7 the contact at $T_{r}$ is removed; and in phase 8 the contact at the $H_{l}$ is removed, being the left toe contact modelled by constraining the two displacements of point $T_{l}$.

\subsection{Muscle modelling in SCI}

Injury to the human spinal cord typically results in complete or partial paralysis of muscles innervated by spinal segments at or below the trauma. The degree of denervation depends on the severity of the SCI. In the C and D levels of AIS, the motor function is preserved below the neurological level (lowest segment where motor and sensory functions are normal), being the difference between $\mathrm{C}$ and $\mathrm{D}$ the muscle activity grade of the key muscular groups (subjects with AIS level C present lower muscle activity than those with AIS level D). The muscle activity grade ranges from 0 (total paralysis) to 5 (active movement, full range of motion, normal resistance).

Both innervated (functional) and partially denervated muscles are modeled as Hill-type actuators. The Hill-type muscle-tendon model [23, 24], which is shown in Fig. 4, consists of a contractile element $(\mathrm{CE})$ that generates the force, a nonlinear parallel elastic element $(\mathrm{PE})$, representing the stiffness of the structures in parallel with muscle fibers, and a nonlinear series elastic element (SE) that represents the stiffness of the tendon which is serially attached to the muscle and completes the muscle-tendon unit. In this model, the pennation angle does not remain constant during muscle fibers contraction. In particular, it increases when the muscle fibers shorten.

(a)

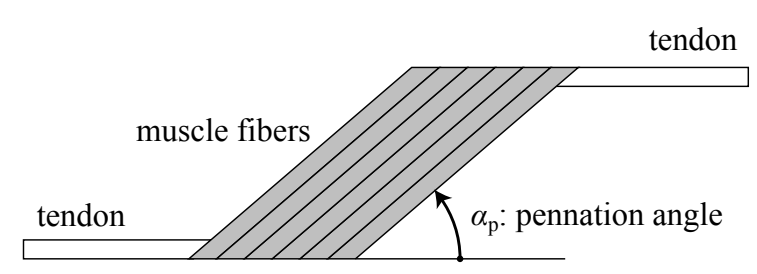

(b)

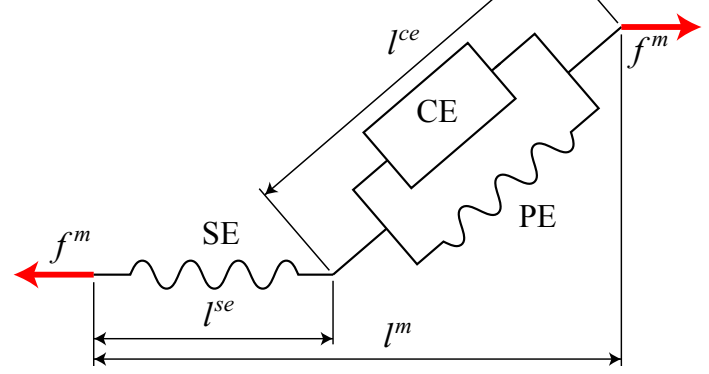

Figure 4: Muscle model: (a) Conceptual scheme; (b) Components of Hill's muscle model [24].

The two differential equations that govern the muscle dynamics are

$$
\begin{gathered}
\dot{a}=h(u, a) \\
\dot{f}^{m}=g\left(a, f^{m}, l^{m}, v^{m}\right)
\end{gathered}
$$

The first equation is the activation dynamics equation that relates muscle excitation $u$ from the central nervous system to muscle activation $a \in[0,1]$. The activation dynamics can be described according to Nagano and Gerritsen [25] by means of the first order differential equation

$$
\dot{a}=(u-a)\left(t_{1} u-t_{2}\right)
$$


where $t_{2}=1 / t_{d}$ and $t_{1}=1 /\left(t_{a}-t_{2}\right)$, being $t_{a}$ and $t_{d}$ the activation and deactivation time constants. Notice that if the activation and its time derivative are known, it is possible to calculate the neural excitation from Equation (8) by solving a quadratic equation.

Eq. (7) defines the force-generation properties as a function of the muscle-tendon length $l^{m}$ and velocity $v^{m}$. The force generated by the CE, $f^{c e}$, is function of the activation $a$, the CE length $l^{c e}$, and its contraction velocity $v^{c e}$. For a detailed description of these relationships the reader is referred to Ackermann [6]. The tendon (SE) can be modeled by a simple quadratic force-strain curve depending on the tendon stiffness [26], see Appendix B. All the values of healthy muscle parameters are obtained from [6].

In this work, the weakness of denervated muscles is modeled through a weakness factor that limits the maximum neural excitation of those muscles. That is, to account for the limited force capacity of a partially denervated muscle the neural excitation will be bounded in the interval $\left[0, u_{\text {lim }}\right]$, what means that the neural excitation of the muscle might never be larger than $u_{\text {lim }}$. As a consequence, the maximum force exerted by the muscle will never reach the value $f_{\max }^{m}$ since the muscle activation, $a$, is always less than or equal to the neural excitation, $u$, see Appendix A for details on the muscle force evaluation. In order to have a compact representation of the degree of injury of a certain individual, the following vector is utilized

$$
\boldsymbol{p}=\left[u_{\text {lim }}^{I L P S O}, u_{\text {lim }}^{R F}, u_{\text {lim }}^{G L U}, u_{\text {lim }}^{H A M S}, u_{\text {lim }}^{V A S}, u_{\text {lim }}^{G A S}, u_{\text {lim }}^{T A}, u_{\text {lim }}^{S O L}\right]^{T}
$$

where $u_{l i m}^{k}$ is the limit value of the neural excitation of muscle $k$, being $k=I L P S O, R F, G L U$, $H A M S, V A S, G A S, T A$ or $S O L$. Note that a value of 1 for $u_{\text {lim }}^{k}$ means that the muscle in fully innervated. In Equation (9) and hereafter, SOL stands for Soleus, TA for Tibialis anterior, GAS for Gastrocnemius, VAS for Vastii, RF for Rectus femoris, HAMS for Hamstrings, GLU for Gluteus, and ILPSO for Ilipsoas. In Alonso et al. [27], a similar approach is used to constrain the muscle force capacity. However, in that research work the muscle force capacity is limited by constraining the maximum activation of denervated muscles instead of their maximum neural excitation. As proposed in the present paper, taking into account the activation dynamics of denervated muscles by constraining the maximum neural excitation may be more adequate for spinal cord injured subjects. Alternatively, Hincapie et al. [28] limit the muscle force capacity using a variable called maximum relative muscle force, which is also between 0 and 1 , that scales the maximum force of the muscles affected by the injury with respect to the able-bodied muscle forces. The approach followed in the present paper may not be usable in particular cases like in stroke patients, where activation dynamics changes after a process of neural reorganization, see Sober et al. [29].

\subsection{Muscles actuating the multibody model}

The muscle groups selected for this research are based on Ackermann [6] and are summarized in Table 2. All the corresponding parameters are estimated for a subject with a height of $1.79 \mathrm{~m}$ and a weight of $73 \mathrm{~kg}$. 


\begin{tabular}{lcccccccccr}
$\begin{array}{l}\text { Muscle } \\
\text { group }\end{array}$ & $\begin{array}{c}f_{\max }^{m} \\
{[\mathrm{~N}]}\end{array}$ & \begin{tabular}{c}
$l_{\text {opt }}^{\text {ce }}[\mathrm{m}]$ \\
\hline \hline ILPSO
\end{tabular} & $\begin{array}{c}l_{\text {slack }} \\
{[\mathrm{m}]}\end{array}$ & $\begin{array}{c}\alpha_{p} \\
{\left[{ }^{\circ}\right]}\end{array}$ & $\begin{array}{c}r_{H \beta} \\
{[\mathrm{cm}]}\end{array}$ & $\begin{array}{c}r_{K \beta} \\
{[\mathrm{cm}]}\end{array}$ & $\begin{array}{c}r_{A \beta} \\
{[\mathrm{cm}]}\end{array}$ & $\begin{array}{c}l_{0}^{m} \\
{[\mathrm{~cm}]}\end{array}$ & $\begin{array}{c}f t \\
{[\%]}\end{array}$ & $\begin{array}{r}\text { width } \\
{[]}\end{array}$ \\
RF & 663 & 0.081 & 0.142 & 7.5 & -5.00 & 0 & 0 & 24.8 & 50 & 1.298 \\
GLU & 1705 & 0.200 & 0.157 & 3.0 & 6.20 & 0 & 0 & 27.1 & 45 & 0.625 \\
HAMS & 1770 & 0.104 & 0.334 & 7.5 & 7.20 & 3.40 & 0 & 38.3 & 35 & 1.197 \\
VAS & 7403 & 0.093 & 0.223 & 4.4 & 0 & -4.30 & 0 & 27.1 & 50 & 0.627 \\
GAS & 1639 & 0.055 & 0.420 & 14.3 & 0 & 2.00 & 5.30 & 48.7 & 50 & 0.888 \\
TA & 1528 & 0.082 & 0.317 & 6.0 & 0 & 0 & -3.70 & 40.6 & 25 & 0.442 \\
SOL & 3883 & 0.055 & 0.245 & 23.6 & 0 & 0 & 5.30 & 28.4 & 20 & 1.039 \\
\hline
\end{tabular}

Table 2: Muscle group properties, being $f_{\max }^{m}$ the maximum muscle force, $l_{o p t}^{c e}$ the optimal length of the CE, $l_{\text {slack }}$ the tendon length, $\alpha_{p}$ the pennation angle, $r_{H \beta}$ the moment arm around the hip joint, $r_{K \beta}$ the moment arm around the knee joint, $r_{A \beta}$ the moment arm around the ankle joint, $l_{0}^{m}$ a parameter used to measure the muscle length, $f t$ the percentage of fast twitch fibers and width a parameter required to evaluate the $\mathrm{CE}$ force.

From the data in Table 2, the length of the different muscles of the right leg is calculated as follows:

$$
l^{m}=l_{0}^{m}-r_{h \beta} \beta_{13}-r_{k \beta} \beta_{34}-r_{a \beta} \beta_{45}
$$

where $l^{m}$ is the length of muscles of the legs, and $\beta_{13}, \beta_{34}$ and $\beta_{45}$ are the joint angles shown in Fig. 2.

In this work, the contraction dynamics is solved to obtain the values of the muscle activation, $a$, since they are involved in the energy expenditure according to the model proposed by Umberger et al. [30]. Then, the activation, $a$, and its time derivative, $\dot{a}$, are used to find the neural excitation, $u$, see Section 3.1. The neural excitations are required for two reasons: they are involved in the calculation of the muscle energy expenditure and they are involved in some of the nonlinear constraints of the optimization procedure since their values must be within the interval $\left[0, u_{l i m}\right]$. Figure 5 shows a flow diagram summarizing the inversion of the contraction and activation dynamics [7].

\subsection{Parameterization of time histories}

The procedure used in this research avoids the forward integration by using parameterization of the time histories of the generalized coordinates by means of spline polynomials and by searching for their optimum values at certain nodal positions. Spline functions have many possibilities that can be used to improve the efficiency of the procedure. In fact, it is easy to have access to the analytical derivatives of the parameterized function, avoiding numerical differentiation. In addition, the interpolation can be splitted into two parts: a more computationally expensive one that can be done in a pre-processing stage and another computationally lighter one that is done during the optimization.

In this work, fifth order splines with periodic boundary conditions are used to parameterize muscle forces, knee motor torques and generalized coordinates. All generalized coordinates are periodic except for coordinate $x_{I 1}$ of the HAT, which is assumed to be the sum of a linear (constant velocity) motion and a periodic oscillation that is also parameterized. The linear motion is function of the average forward velocity and the initial value of coordinate $x_{I 1}$, being both fixed during optimization. According to García-Vallejo and Schiehlen [7], the periodicity of the set of points used to calculate the interpolating polynomials is reinforced. In the case of fifth 


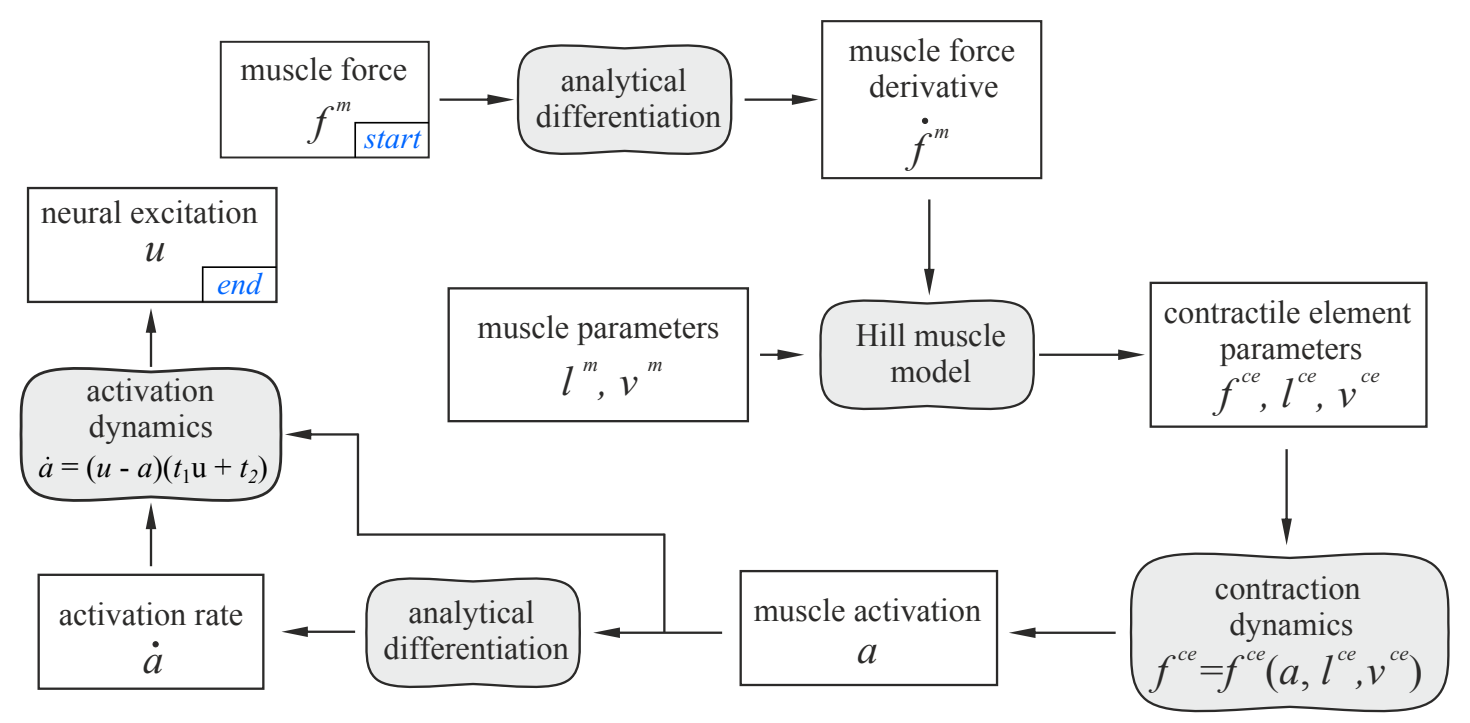

Figure 5: Flow diagram of the inversion of the activation and contraction dynamics. The inversion process starts from the muscle force value and ends at the neural exitation evaluation.

order periodical splines, the degree of periodicity is defined according to

$$
\begin{aligned}
& f_{1}=f_{N} \\
& f_{1}^{\prime}+O\left(h^{3}\right)=f_{N}^{\prime}+O\left(h^{3}\right) \\
& f_{1}^{\prime \prime}+O\left(h^{3}\right)=f_{N}^{\prime \prime}+O\left(h^{3}\right) \\
& f_{1}^{\prime \prime \prime}+O\left(h^{3}\right)=f_{N}^{\prime \prime \prime}+O\left(h^{3}\right)
\end{aligned}
$$

where $f_{1}, f_{1}^{\prime}, f_{1}^{\prime \prime}$ and $f_{1}^{\prime \prime \prime}$ are the values of the function to be interpolated at the first point node and its first, second and third derivatives, respectively, and $h$ is the distance between points. Using Taylor series expansions, it is possible to find the derivatives at the first node by using a backward difference formula and the derivatives at the last node, $N$, by using a forward difference formula. Then, Equations (11) result in a system of four linear equations from which it is possible to obtain the values of $f_{1}, f_{2}, f_{N-1}$ and $f_{N}$ that improve the periodicity of the data set to be interpolated.

\section{Parameter Optimization}

The simulation of human walking motion is now treated as a large parameter optimization problem. The optimization parameters, also called design variables, are used to reconstruct the muscle force histories and the generalized coordinate histories of a walking cycle as well. Such a set of parameters are found by minimizing a cost function which is evaluated based on energetic and aesthetic reasons. Finally, the motion and muscle forces time histories reconstructed from the optimization parameters are asked to fulfill the equations of motion of the multibody system, the kinematic constraints as well as other physical and physiological relations.

The complete set of design variables are summarized in vector $\boldsymbol{\chi}$. Assuming the usage of a number of $n_{n}$ nodes for parameterization of time histories, this vector is itself built from six different kinds of vectors as follows:

1. A set of $n_{c}$ vectors, each one containing all nodal values of a single generalized coordinate. Thus, one of those $n_{c}$ vectors may be denoted as $\boldsymbol{y}_{i}, i=1,2, \ldots n_{c}$. Therefore, the total number of design variables due to trajectory parameterization will be $n_{c} \times n_{n}$. 
2. A set of $N_{m}$ vectors, each one containing all nodal values of a single muscle force. Thus, one of those $N_{m}$ vectors may be denoted as $\boldsymbol{f}_{j}^{m}, j=1,2, \ldots N_{m}$. Therefore, the total number of design variables due to muscle force parameterization will be $N_{m} \times n_{n}$.

3. Two vectors $\boldsymbol{T}_{k r}$ and $\boldsymbol{T}_{k l}$ containing all nodal values of the motor torques at the right and left knees, respectively. Therefore, the total number of design variables due to motor torque parameterization will be $2 \times n_{n}$.

4. A vector with eight components representing the durations of the eight phases of a walking cycle $\boldsymbol{t}_{p h}$.

5. A vector with geometrical parameters describing the kinematic constraints of the feet on the ground $\boldsymbol{p}_{g}$.

6. A vector containing design parameters of the orthosis $\boldsymbol{p}_{o}$.

According to the previous explanation, the vector of design variables can be written as:

$$
\chi=\left[\begin{array}{lllllllllll}
\boldsymbol{y}_{1}^{T} & \ldots & \boldsymbol{y}_{n_{c}}^{T} & \boldsymbol{f}_{1}^{m T} & \ldots & \boldsymbol{f}_{N_{m}}^{m}{ }^{T} & \boldsymbol{T}_{k r}^{T} & \boldsymbol{T}_{k l}^{T} & \boldsymbol{t}_{p h}^{T} & \boldsymbol{p}_{g}^{T} & \boldsymbol{p}_{o}^{T}
\end{array}\right]^{T}
$$

with

$$
\begin{aligned}
& \boldsymbol{t}_{p h}=\left[t_{1}, t_{2}, \ldots t_{8}\right]^{T} \\
& \boldsymbol{p}_{g}=\left[L_{r}, L_{l}\right]^{T} \\
& \boldsymbol{p}_{o}=\left[T_{a r}^{0}, k_{a r}, T_{a l}^{0}, k_{a l}\right]^{T}
\end{aligned}
$$

where $L_{r}$ and $L_{l}$ are the right and left step lengths, see Fig. 3.

\subsection{Optimization framework}

Minimizing energy expenditure during walking is a reasonable criteria that the central neural system may use when dealing with muscles recruitment, specially when walking long distances. For this reason, it makes sense to obtain muscle forces and generalized coordinates by minimizing the metabolical cost of walking. In this investigation, the energy expenditure model due to Umberger et al. [30] is used as measure of the metabolical cost. This energy measure was also used by Ackermann [6] while other authors have used different cost functions as for example a measure of the muscle fatigue, see Brand et al. [31] and Peasgood et al. [1].

Umberger et al. [30] provided a measure of the metabolical expenditure including thermal and mechanical energy liberation rates during simulated muscle contractions of mammalians at normal body temperature. According to their model, the total energy rate of a single muscle is written as follows:

$$
\dot{E}=\dot{E}\left(l^{c e}, v^{c e}, f^{c e}, a, u, \boldsymbol{p}_{m}\right)
$$

where $l^{c e}$ is the $\mathrm{CE}$ length, $v^{c e}$ is the $\mathrm{CE}$ velocity, $f^{c e}$ is the CE force, $a$ is the muscle activation, $u$ is the neural excitation and $\boldsymbol{p}_{m}$ is a vector summarizing all muscle constant parameters required to evaluate the energy rate, see Umberger et al. [30]. The previous expression of the energy rate can be integrated in time in order to obtain the amount of energy spent during walking as

$$
E=\int_{t_{0}}^{t_{f}} \dot{E}\left(l^{c e}, v^{c e}, f^{c e}, a, u, \boldsymbol{p}_{m}\right) \mathrm{d} t
$$

where $t_{0}$ and $t_{f}$ represents the initial and final times of the gait cycle, respectively.

A more meaningful measure of energy consumption when considering walking long distances in normal conditions is the energy expended per unit of length what can be obtained by dividing the 
total energy of one cycle by the distance walked. This is called the total energy of transportation and reads as

$$
E^{t}=\frac{E}{L_{r}+L_{l}}
$$

Since the time histories of the muscle forces and of the generalized coordinates are obtained by optimization techniques trying to minimize the energy consumption there is a need to follow a certain motion pattern. Otherwise, in an attempt to reduce the energy expenditure a non-logical solution could be found. Therefore, a measured walking motion is used to force the model to follow a certain motion. This fact has some other advantages in designing orthosis. First, the simulated motion of an individual wearing an orthosis should be close to normal walking patterns which is desirable for aesthetical reasons. Second, the simulated contact forces will be close to those of a normal walking cycle what would result in non-significant modification of the contact forces at non-damaged feet. This is reasonable in case of non-severe damages since other aspects like pain may be more important than enforcing a symmetric walking motion.

The deviation with respect to normal walking patterns is evaluated as follows:

$$
J_{\text {dev }}=\int_{t_{0}}^{t_{f}} \sum_{i=1}^{n_{x}} \frac{\left(x_{i}(t)-x_{i}^{m}(t)\right)^{2}}{\sigma_{i}^{2}} \mathrm{~d} t
$$

where $x_{i}$ is a time dependent variable of the model and $x_{i}^{m}$ refers to the experimentally measured value of the same variable. These variables, $x_{i}$ with $i=1,2, \ldots n_{x}$, include the generalized coordinates and ground reaction forces, being $n_{x}$ the number of nodal values of generalized coordinates and ground reaction forces. In (17), $\sigma_{i}$ is a characteristic measure of the time variability of $x_{i}$. Dividing by $\sigma_{i}$ the differences between measured and simulated values of all $x_{i}$ are scaled. In this investigation, the mean square deviation with respect to the mean is used as a measure of the time variability as

$$
\sigma_{i}=\sqrt{\frac{1}{T} \int_{t_{0}}^{t_{f}}\left(x_{i}(t)-X_{i}\right)^{2} \mathrm{~d} t} \quad \text { with } \quad X_{i}=\frac{1}{T} \int_{t_{0}}^{t_{f}} x_{i}(t) \mathrm{d} t .
$$

where $X_{i}$ is the mean of $x_{i}(t)$ in the measured walking cycle. The measured motion used in this research was obtained by Ackermann and Gros [32] by measuring the walking motion of a subject wearing sport shoes and walking at his preferred velocity.

When designing an active assisting device as the SCKAFO considered in this paper, it makes sense to consider the power consumption of the actuators, see Ref. [27]. For this reason, the objective function of this optimization problem is augmented by including two measures of the actuator performance. The power consumption is evaluated based on the root mean square (RMS) of the mechanical power developed during the whole gait cycle $T$. Thus, the following function is used:

$$
P_{R M S}=\sqrt{\frac{1}{T} \int_{t_{0}}^{t_{f}}\left(T_{k r} \cdot \dot{\beta}_{34}\right)^{2} \mathrm{~d} t}
$$

where only the RMS power developed by the actuator in the right leg has been included since the model considered in this research is fully symmetrical and including the RMS of the left leg would add no extra information to the objective function. Another important measure of the actuator performance is the torque exerted by the motor. In this respect, a measure of the RMS actuation torque has been also considered. The RMS torque is evaluated as follows:

$$
T_{R M S}=\sqrt{\frac{1}{T} \int_{t_{0}}^{t_{f}}\left(T_{k r}\right)^{2} \mathrm{~d} t}
$$

where again the symmetry of the model is recalled to use only the RMS of the right leg actuator torque. 
Based on the previous definitions, three different cost functions are considered in this research. In the first one, the value of the cost function is calculated only using the metabolical cost of transportation, $E^{t}$, and the measure of the deviation from normal walking patterns, $J_{d e v}$, as follows:

$$
f_{A}=\omega_{E} \frac{E^{t}}{E_{0}^{t}}+\omega_{J} J_{d e v}
$$

where $E^{t}$ is divided by a reference value, $E_{0}^{t}$, to obtain a non-dimensional magnitude of the same order of $J_{d e v}$ for balancing of the two terms of the third cost function (23) to get comparable numbers, and $\omega_{E}$ and $\omega_{J}$ are two weighting factors.

The second cost function is formulated by including the RMS mechanical power of the actuator as

$$
f_{B}=\omega_{E}\left(\frac{E^{t}}{E_{0}^{t}}+\frac{P_{R M S}}{P_{R M S 0}}\right)+\omega_{J} J_{d e v}
$$

where $P_{R M S}$ is divided by the reference value $P_{R M S 0}$ and the resulting non-dimensional quotient is affected by the same weight factor of the metabolical cost of transportation to emphasize that it is a measure of the multibody model dynamical performance.

Finally, the third cost function is formulated by including the RMS measure of the actuator torque as follows:

$$
f_{C}=\omega_{E}\left(\frac{E^{t}}{E_{0}^{t}}+\frac{T_{R M S}}{T_{R M S 0}}\right)+\omega_{J} J_{d e v}
$$

where again the weight function $\omega_{E}$ affects the metabolical cost and the non dimensional quotient of $T_{R M S}$ and the reference value $T_{R M S 0}$.

It is important to bear in mind that minimizing a measure of the actuator performance is somehow contradictory to minimizing the metabolical cost of transportation, since a minimal contribution of the active orthosis requires a maximal contribution of the muscles and viceversa. In other words, the actuator would work more if the measures of its performance are not included in the cost function. This interesting relation is shown in the numerical results sections with the help of different examples.

\subsection{Constraint formulation}

The solution of the optimization algorithm must fulfill a set of constraints as stated at the beginning of this section. The set of constraints is summarized as follows.

1. In the case of healthy muscles, neural excitations must be bounded in the interval $[0,1]$. In addition, partially denervated muscles must have a neural excitation bounded in the interval $\left[0, u_{l i m}\right]$, where $u_{l i m}$ is the maximum excitation level which is achievable by the muscle. This kind of constraint ensures that muscle forces are consistent with the activation and contraction dynamics of the muscles.

2. Ground clearance must be positive or equal to zero to ensure no penetration of the feet into the ground.

3. Positive normal contact forces to avoid bilateral constraints between the feet and the ground.

4. Tangent contact forces on the feet must be consistent with Coulomb's friction model to avoid foot sliding.

5. The averaged velocity is fixed.

6. Design variables are bounded. These bounds may be due to some physiological reasons like for example the amplitud of the relative motion allowed by a certain joint. 
7. Other physiological constraints that may help to the convergence of the optimization algorithm like for instance constraining the maximal achieved knee flexion during the swing phase or the maximal achieved hip extension during the stance phase, see Ackermann [6].

8. Each knee is locked during the stance phase of the leg it belongs to $\left(\beta_{34}=0\right.$ and $\left.\beta_{67}=0\right)$. Therefore, the motors are not actuating during such periods $\left(T_{k r}=0\right.$ if $\beta_{34}=0$, and $T_{k l}=0$ if $\left.\beta_{67}=0\right)$.

9. The Klenzak ankle joint avoid positive rotation of the ankle and, therefore, $\beta_{45} \leq 0$ and $\beta_{78} \leq 0$.

10. Equations of motion must be fulfilled within a certain tolerance.

11. Kinematic constraints must be fulfilled within a given tolerance.

Exactly satisfying the equations of motion, although it would be desirable, seems to be extremely difficult due to the parameterization of the motion and muscle forces by using splines. Therefore, one have to accept a small violation of the equations of motion. In order to quantify such an infringement, the constraints are formulated in terms of generalized joint torques since their usual range of values are known approximately from inverse dynamics of normal walking. In what follows, the optimization constraints of the equations of motion are formulated.

As explained before, muscle forces as well as generalized coordinates are considered as design variables. Thus, during the iterative solution of the optimization problem one will have generalized coordinates and muscle forces that are not completely consistent. In case a consistent set of muscle forces and generalized coordinates is found, the following system of equation holds

$$
\boldsymbol{M} \ddot{\boldsymbol{y}}+\boldsymbol{k}=\boldsymbol{q}_{a}+\boldsymbol{B} \boldsymbol{A} \boldsymbol{f}^{m}+\boldsymbol{q}_{a c t}+\boldsymbol{q}_{a n k}+\boldsymbol{C}_{p h}^{T} \boldsymbol{\lambda}_{p h}
$$

Then, it is known that there is a unique set of Lagrange multipliers $\boldsymbol{\lambda}_{p h}$ for each phase of the motion that can be calculated by using the pseudo-inverse of the Jacobian matrix of the constraints, see Strang [33], as

$$
\boldsymbol{\lambda}_{p h}=\left(\boldsymbol{C}_{p h}^{T}\right)^{+}\left(\boldsymbol{M} \ddot{\boldsymbol{y}}+\boldsymbol{k}-\boldsymbol{q}_{a}-\boldsymbol{B} \boldsymbol{A} \boldsymbol{f}^{m}-\boldsymbol{q}_{a c t}-\boldsymbol{q}_{a n k}\right)
$$

In case the motion and the muscle forces are not fully consistent, the previous equation provides an estimation of the Lagrange multipliers in a least square sense [33] and for that reason it is denoted as

$$
\boldsymbol{\lambda}_{p h}^{*}=\left(\boldsymbol{C}_{p h}^{T}\right)^{+}\left(\boldsymbol{M} \ddot{\boldsymbol{y}}+\boldsymbol{k}-\boldsymbol{q}_{a}-\boldsymbol{B} \boldsymbol{A} \boldsymbol{f}^{m}-\boldsymbol{q}_{a c t}-\boldsymbol{q}_{a n k}\right)
$$

Due to this inconsistence between the motion and the muscle forces, we have to accept a certain error $\boldsymbol{e}_{m}$ in the equations of motion

$$
\boldsymbol{M} \ddot{\boldsymbol{y}}+\boldsymbol{k}=\boldsymbol{q}_{a}+\boldsymbol{B} \boldsymbol{A} \boldsymbol{f}^{m}+\boldsymbol{q}_{a c t}+\boldsymbol{q}_{a n k}+\boldsymbol{C}_{p h}^{T} \boldsymbol{\lambda}_{p h}^{*}+\boldsymbol{e}_{m}
$$

Then, using Equation (26), Equation (27) and the pseudo-inverse of $\boldsymbol{C}_{p h}^{T}$ one can write

$$
\boldsymbol{\lambda}_{p h}^{*}=\left(\boldsymbol{C}_{p h}^{T}\right)^{+}\left(\boldsymbol{C}_{p h}^{T} \boldsymbol{\lambda}_{p h}^{*}+\boldsymbol{e}_{m}\right) \quad \rightarrow \quad\left(\boldsymbol{C}_{p h}^{T}\right)^{+} \boldsymbol{e}_{m}=\mathbf{0}
$$

It shall be noted here that the number of components of vector $\boldsymbol{e}_{m}$ is equal to the number of generalized coordinates while the number of rows of matrix $\left(\boldsymbol{C}_{p h}^{T}\right)^{+}$is the number of active constraints, $n_{a}$, at the phase $p h$ of motion. In the previous equation, it has been used that $\left(\boldsymbol{C}_{p h}^{T}\right)^{+} \boldsymbol{C}_{p h}^{T}=\boldsymbol{I}$ since, due to the non-redundant set of kinematic constraints used, the columns of $\boldsymbol{C}_{p h}^{T}$ are independent.

In order to ensure the fulfillment of the equations of motion, the error is defined using the generalized force vector associated to joint torques instead of the vector of torques itself. This 
can be done since the terms of the generalized force vector of the joint torques acting on the coordinates describing the absolute motion of the trunk and pelvis with respect to the inertial frame are zero. This way, the generalized force vector of the joint torques can be obtained from muscle forces

$$
\boldsymbol{q}^{m}=\boldsymbol{B} \boldsymbol{A} \boldsymbol{f}^{m}
$$

and from the estimated Lagrange multipliers according to (24) or (26), respectively

$$
\boldsymbol{q}^{*}=\boldsymbol{M} \ddot{\boldsymbol{y}}+\boldsymbol{k}-\boldsymbol{q}_{a}-\boldsymbol{q}_{a c t}-\boldsymbol{q}_{a n k}-\boldsymbol{C}_{p h}^{T} \boldsymbol{\lambda}_{p h}^{*} .
$$

The optimization constraint of the equations of motion is now written as

$$
\left|\boldsymbol{q}_{i}^{m}-\boldsymbol{q}_{i}^{*}\right| \leq \varepsilon_{m} \quad i=1,2, \ldots n_{c},
$$

where $\varepsilon_{m}$ is the tolerance of the constraint satisfaction. The error term to be bounded at each control point is

$$
e_{i, j}(\chi)=\left(\boldsymbol{q}_{i}^{m}-\boldsymbol{q}_{i}^{*}\right)_{j} \quad i=1,2, \ldots n_{c}, \quad j=1,2, \ldots n_{c p}
$$

where $e_{i, j}(\boldsymbol{\chi})$ is a scalar equation representing the violation of the nonlinear constraint associated with equation of motion $i$ at control point $j$, and $n_{c p}$ is the number of control points where constraints must be fulfilled. According to Equation (31), Equation (32) leads to two constraints per control point and per each component of the generalized joint torque vector as follows

$$
\begin{aligned}
& e_{i, j}(\chi) / \varepsilon_{m}-1 \leq 0 \\
& -1-e_{i, j}(\chi) / \varepsilon_{m} \leq 0
\end{aligned}
$$

The formulation used in this research leads to a well posed constrained optimization problem. Using Equations (33) the constraints of the equations of motion are continuous and the Jacobian of the constraints provides good information when searching for the optimum, what results in a reasonable number of iterations of the optimization algorithm.

\section{$5 \quad$ Numerical Results}

Several numerical results are collected in this section, being all of them obtained by using a model of the musculoskeletal system including the orthoses described in Section 3. The mass and moments of inertia of all segments are modified to include the weights and moments of inertia of the different segments of the active orthosis. The positions of the centers of mass are accordingly recalculated.

For the optimizations carried out in this numerical section, the SQP algorithm included in Matlab $^{\circledR}$ fmincon subroutine has been used. In all the cases, the termination tolerances for the SQP optimization algorithm were fixed to TolFun $=10^{-3}$, TolCon $=10^{-3}$ and TolX $=10^{-4}$, being TolFun the termination tolerance for the cost function, TolCon the termination tolerance for the constraints violation and $\operatorname{Tol} X$ the termination tolerance for design variables vector. In all simulations, 29 nodes have been used for the spline parameterization while 80 control points have been used to check the fulfillment of the constraints. The tolerances for the fulfilment of the equations of motion and of the kinematical constraints were $\varepsilon_{m}=2 \mathrm{Nm}$ and $\varepsilon_{k}=5 \mathrm{~mm}$, respectively. The reference values $E_{0}^{t}, P_{R M S 0}$ and $T_{R M S 0}$ are fixed to 100,1 and 1, respectively, based on the order of magnitude obtained for $E^{t}, P_{R M S}$ and $T_{R M S}$ in some initial tentative simulations.

This section is divided in four parts. The first one contains the results of the gait simulation of a spinal cord injured individual including a comparison with normal gait patterns. The second part deals with a comparison of the different cost functions described previously in Section 4.1. The third part includes a comparison of the dynamics of three different levels of injured individuals wearing an orthosis and the last part deals with the stiffness requirements of the flexible ankle depending on the level of injury. 


\subsection{Simulation of an incomplete SCI case}

The objective of this section is to show the performance of the optimization framework described previously in the case of simulating the gait of an injured subject who is wearing the active orthosis described before. For the simulation, it will be assumed that the subject is walking in a steady state in which the gait cycle is fully periodical. The level of injury of the subject is represented with the help of a vector of neural excitation limits as described in Section 3.1. Thus, the injury of the individual is represented as follows:

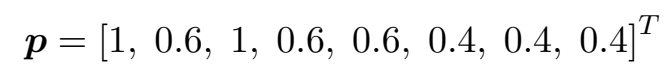

where the excitation of mono-articular hip muscles are not limited since it is assumed that the target patients that may use the orthosis preserve motor function of the hip muscles. According to the injury vector in Equation (34), muscles Iliopsoas and Gluteus are fully innervated while the rest are partially denervated, being the injury more severe for the lower muscles of the leg.

Figure 6 shows the time histories of the different generalized coordinates of the model defined in Figure 2 along with the values of such coordinates in the reference motion used as normal pattern. In general terms, the simulated motion follows the reference one. It can be seen that the distance walked in the simulated gait cycle is smaller than the one of the reference motion, what is expectable since the reference motion correspond to a healthy individual. The amplitude of the vertical oscillation of the center of mass of the pelvis-trunk body is significatively smaller in the simulated motion. Checking the generalized coordinates describing the relative rotation of the knees it can be observed how the knee locking constraints are active during the stance phases. In addition, the rotation of the ankle is never positive due to the kinematic constraints related to the Klenzak ankle joint.

A look at the ground contact forces in Figure 7 reveals some differences with respect to the reference pattern. The tangent contact force at the beginning of the cycle has opposite sign to the reference one while its amplitude is smaller as well. This means that the foot tends to move slightly backwards after the heel strike. This effect may be related to the foot-ground contact model where it was assumed that the velocity of the foot is null at the instant of contact. After the first instants the tangent force tries to follow the experimental pattern as shown in Figure 7. With respect to the normal contact force, the agreement is remarkable. As shown in the figure, the simulated normal ground contact forces show some fluctuations with respect to the reference ones.

Figure 8 shows the neural excitation along with the muscle activations for the sixteen muscles of the simulated model. As expected, the muscle activation follows with some delay the pattern of the neural excitation. The delay depends on the constant $t_{1}$ and $t_{2}$ of each muscle as explained in Section 3.1. It can clearly be seen how the neural excitation is limited for several muscles according to the injury vector in Equation (34). Even if the possible activation is limited for the lower leg muscles, due to the contribution of the actuation knee torques and the Klenzak ankle joints, the motion of the subject is feasible. 


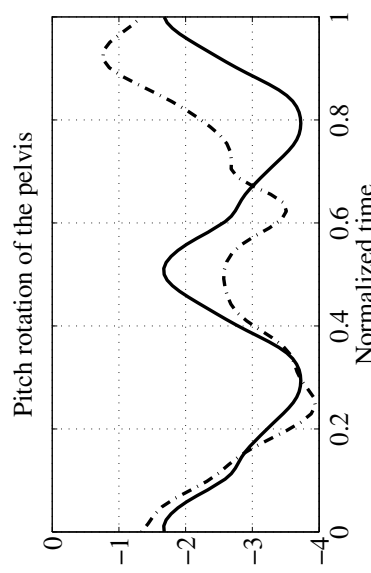

(o) II

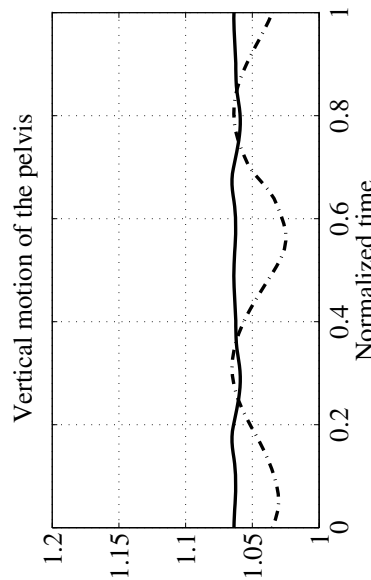

(u) ${ }^{\mathrm{II}} \mathrm{z}$

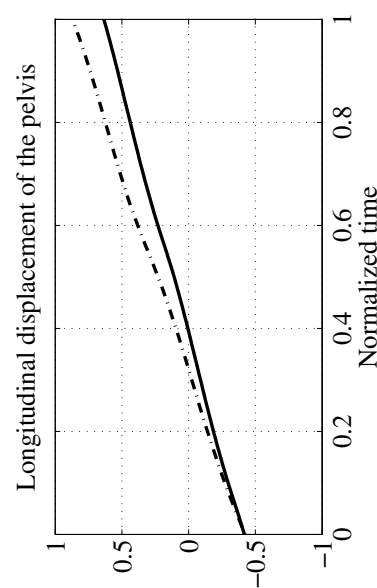

(ui) ${ }^{\mathrm{II}} \mathrm{x}$

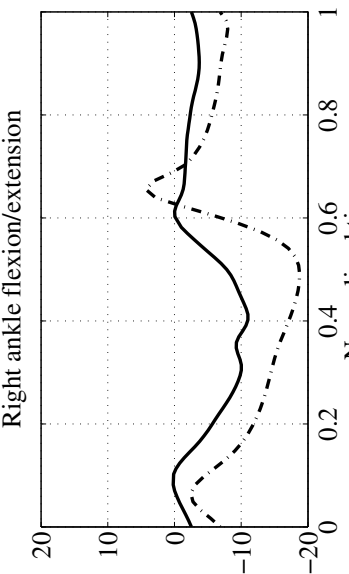

(.) ${ }^{\text {st }} \mathrm{g}$

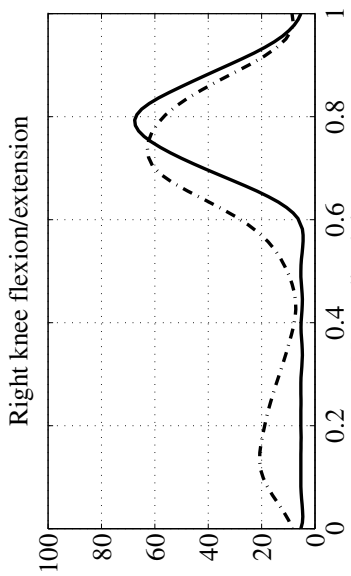

$\left.{ }_{(0)}\right)^{t \varepsilon} g$

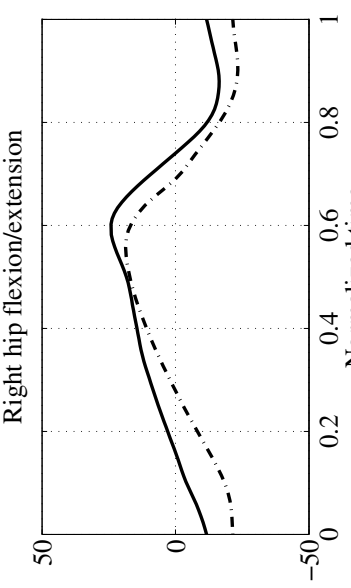

${ }_{(0)}^{\varepsilon I} \mathrm{~g}$

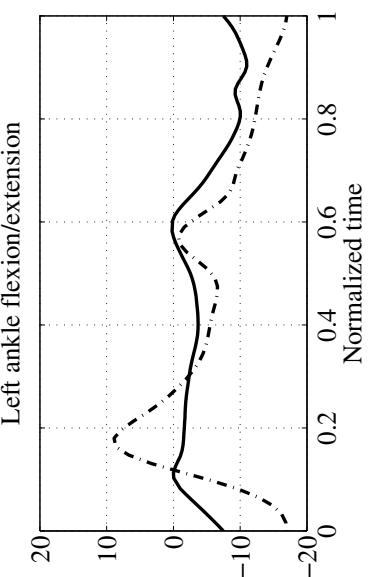

$\left(0^{8 L} \mathrm{~g}\right.$

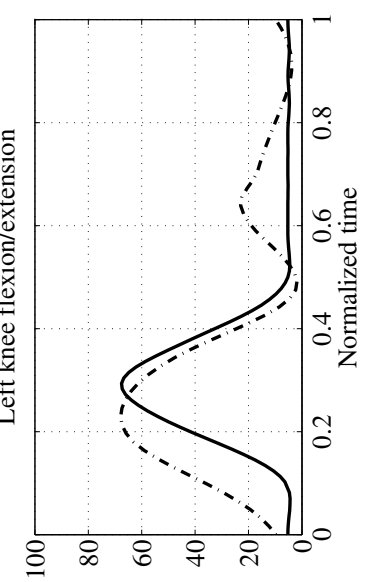

$\left({ }^{(0)}{ }^{L 9} \mathrm{~g}\right.$

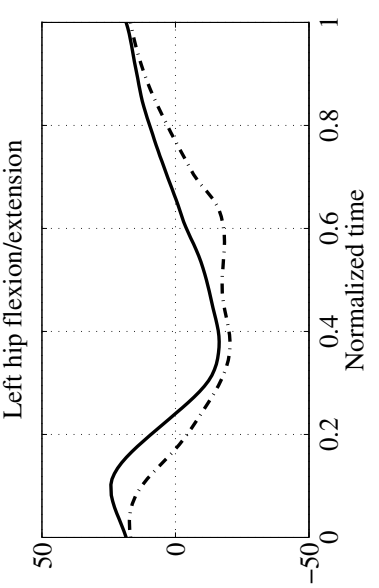

$\left(0^{91} \mathrm{~d}\right.$

Figure 6: Trajectories of the generalized coordinates of the model depicted in Fig. 2 for the reference gait motion (dash-dotted line) and for the simulated gait motion (solid line). 

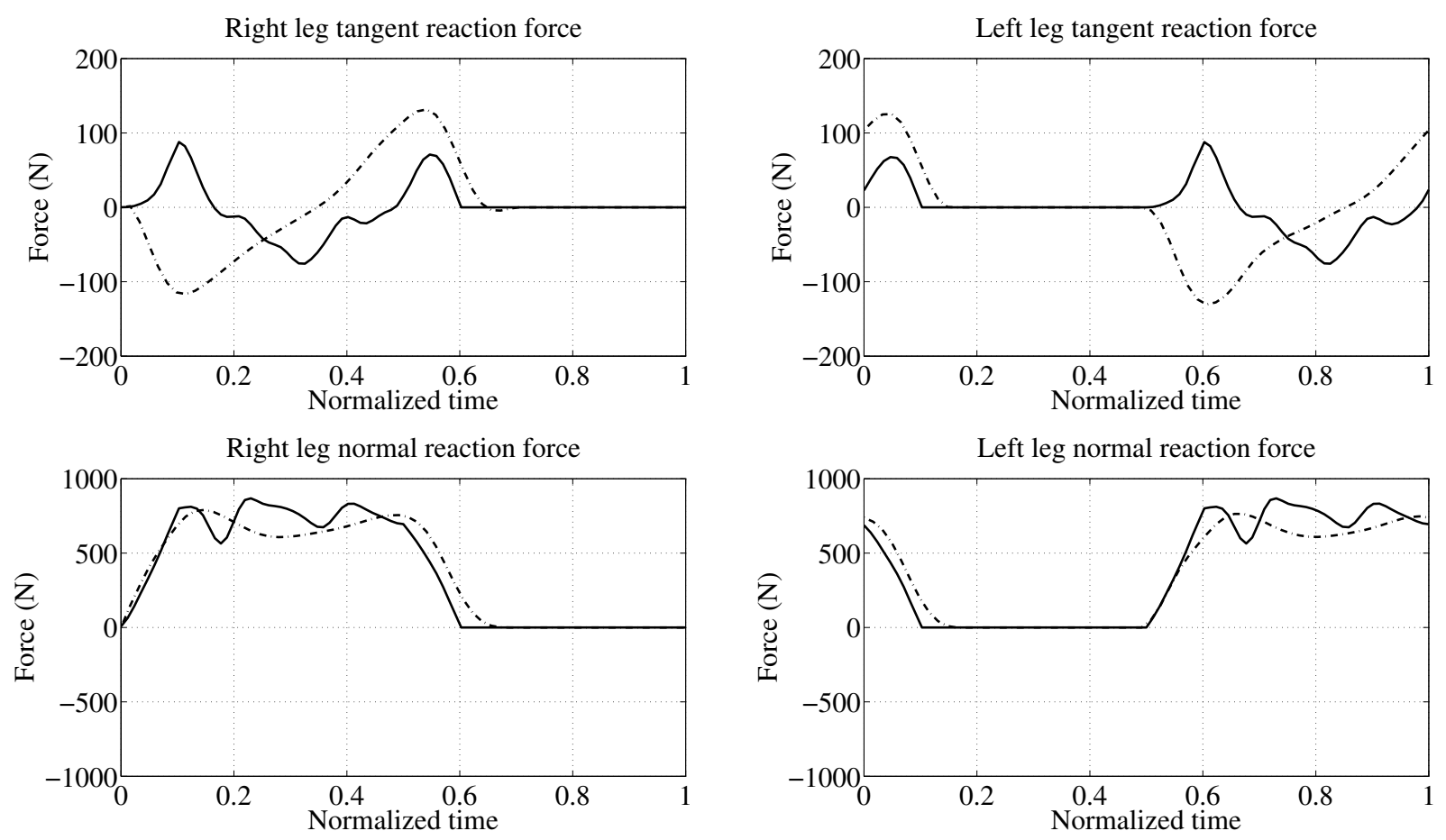

Figure 7: Ground reaction forces for the reference gait motion (dash-dotted line) and for the simulated gait motion (solid line). The upper left and right plots show the tangent ground reaction forces of the right and left feet, respectively. The lower left and right plots show the normal ground reaction forces of the right and left feet, respectively. 

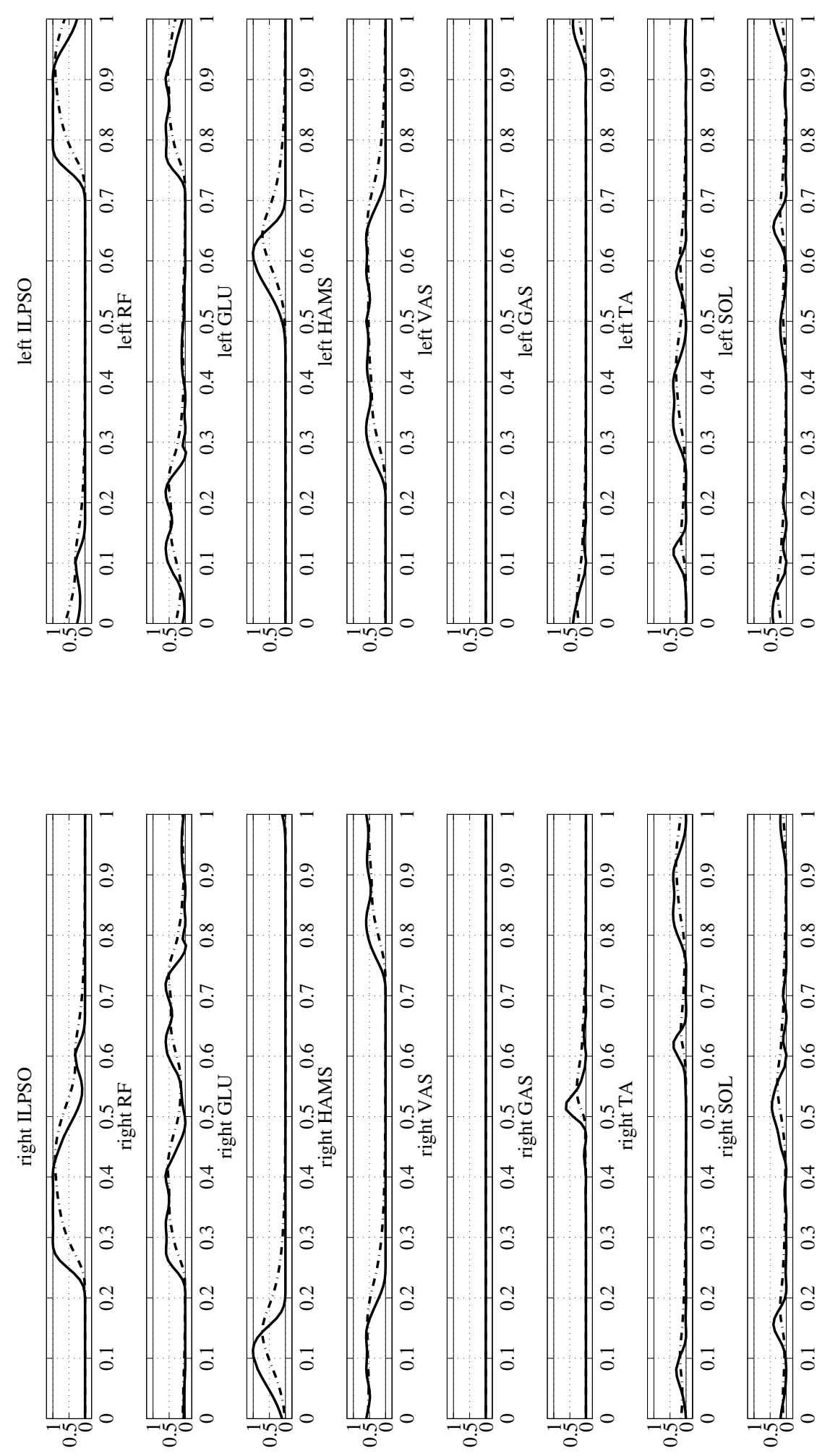

Figure 8: Neural excitations (solid line) and muscle activation (dash-dotted line) for the sixteen muscles of the model depicted in Fig. 2 according to the definition by Eq. (9). The eight plots in the top part correspond to the muscles of the left leg while the eight plots on the bottom part correspond to the muscles of the right leg. 


\subsection{Influence of the cost function on the injured individual gait performance}

In this section, the parameters of the subject represented by the vector in Equation (34) are used to test the different cost functions described in Section 4.1. The weight factors $\omega_{E}$ and $\omega_{J}$ are both set to 1 . The metabolical cost of transportation obtained are $274.67 \mathrm{~J}, 341.16 \mathrm{~J}$ and 328.11 $\mathrm{J}$ for cost functions $f_{A}, f_{B}$ and $f_{C}$, respectively. As expected, using the cost function $f_{A}$, the one which does not include any performance measure of the orthosis, the metabolical cost is the least. It means that the active orthosis is contributing to motion as much as it is required. Using the cost functions $f_{B}$ and $f_{C}$, the contribution of the active orthosis to the gait is minimized what results in an increase of the metabolical energy expenditure. Regarding the deviation from the reference gait pattern, $J_{d e v}$, values of $12.9,12.9$ and 13.1 for cost functions $f_{A}, f_{B}$ and $f_{C}$, respectively, are found.
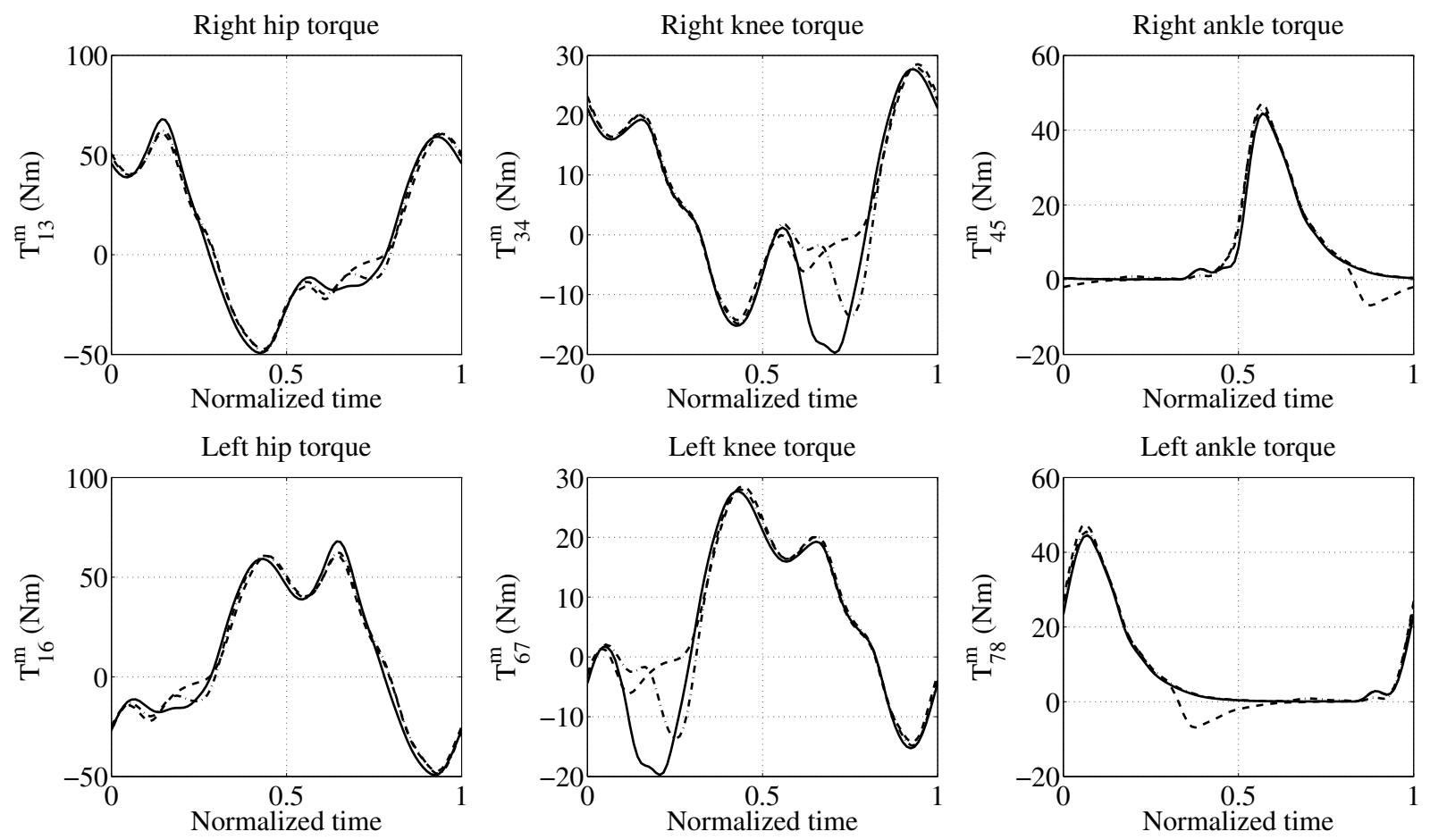

Figure 9: Muscle joint torques obtained using cost function $f_{A}$ (solid line), cost function $f_{B}$ (dashed line) and cost function $f_{C}$ (dash-dotted line). The upper row of plots represents the muscle joint torques in the right leg joints while the lower row of plots represents the muscle joint torques in the left leg joints. From left to right, each row shows the muscle joint torques at the hip, knee and ankle joints.

Figure 9 shows the time histories of the net muscle torques obtained using the three different cost functions. Note that the net muscle torques are very similar in a wide part of the walking cycle and separate one from each other when the motors actuate at the knees. This can be seen in Figure 10, where the motor torques at the right and left knees are shown. It is interesting how the motor torques are large when using the function $f_{A}$ since the motor performance is not included in the cost function in any sense. On the other hand, including the motor performance in cost functions $f_{B}$ and $f_{C}$ leads to smaller values of the motor torques. In respect to the flexible ankle, it can be seen that the cost function does not influence significatively the net torque at the ankle joint as shown in Figure 10.

Figure 11 shows the total torques at the knees and ankles obtained as the sum of the torques exerted by the active orthosis and by the different muscles at each joint. In general, the three patterns are very similar. This fact seems to be logical since the motion obtained is very similar 

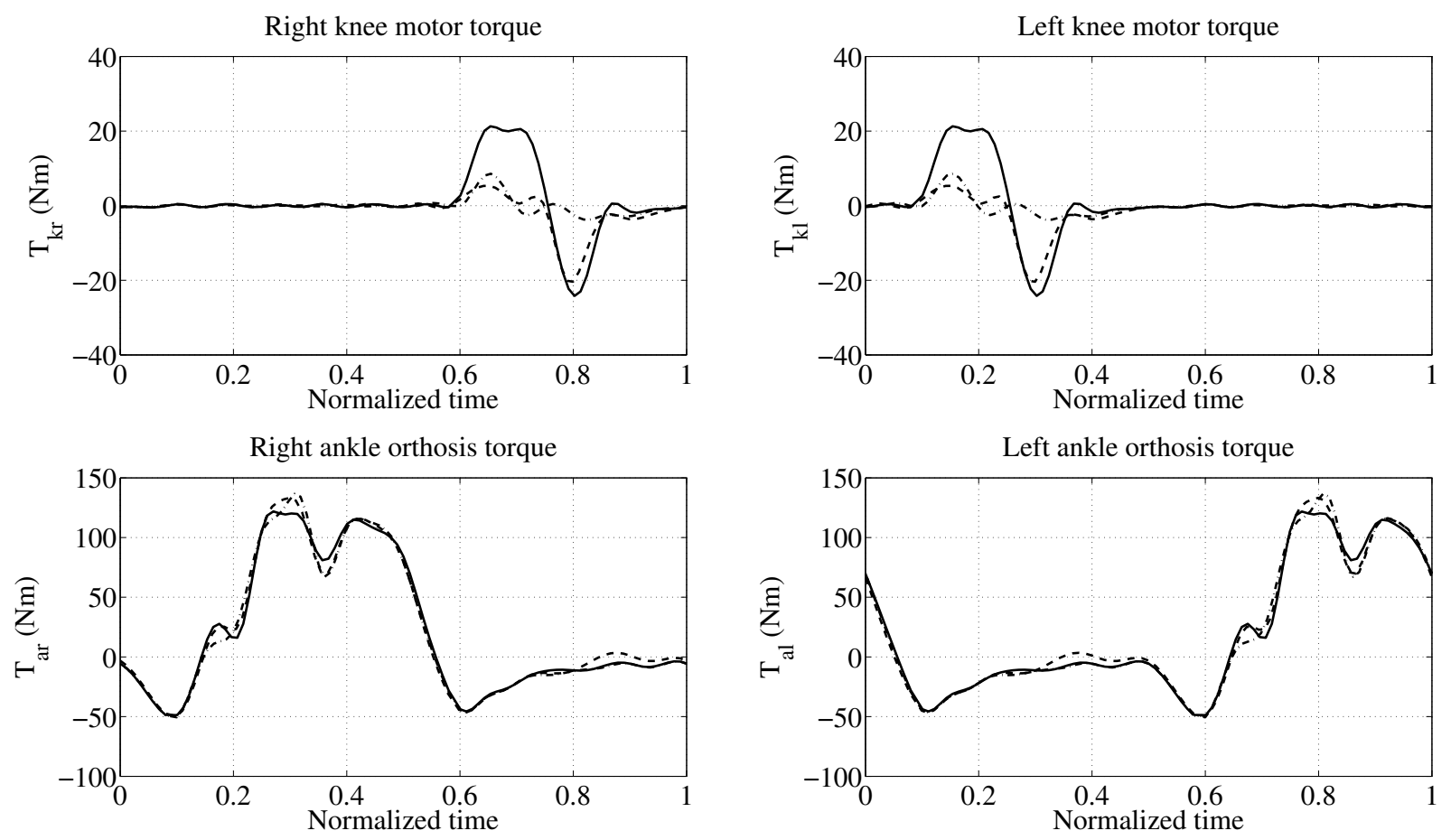

Figure 10: Orthosis joint torques obtained using cost function $f_{A}$ (solid line), cost function $f_{B}$ (dashed line) and cost function $f_{C}$ (dash-dotted line). The upper row of plots represents the torques exerted by the orthosis motors at the knee joints of the model while the lower row of plots represents the torques exerted by the orthosis flexible ankle at the ankle joints of the model.

in all cases as shown by the values of $J_{d e v}$ previously reported.

\subsection{Comparison of gait patterns corresponding to three cases of incomplete SCI}

In this section, three cases of SCI have been simulated: two subjects with incomplete SCI with AIS level C and another with AIS level D. In both AIS levels C and D, the motor function is preserved below the neurological level. The difference between those levels is the number of key muscles below the neurological level that have a muscle activity grade less than 3. In AIS level $\mathrm{C}$, more than half of key muscles below the neurological level have a muscle grade less than 3 . In AIS level D, at least half of key muscles below the neurological level have a muscle grade of 3 or more. Thus, the following vectors of weakness factors are defined according to Equation (9) to simulate the three subjects:

1. Case 1: AIS D subject: $\boldsymbol{p}=[1,0.6,1,0.6,0.6,0.4,0.4,0.4]^{T}$.

2. Case 2: AIS C subject: $\boldsymbol{p}=[1,0.2,1,0.2,0.2,0.2,0.2,0.2]^{T}$.

3. Case 3: AIS C subject: $\boldsymbol{p}=[1,0.2,1,0.2,0.2,0.2,0.0,0.0]^{T}$.

For the simulations included in this section, the cost function denoted as $f_{A}$ has been used, in which the motor performance is not included in any sense. The metabolical costs of transportation obtained are $274.67 \mathrm{~J}, 316.33 \mathrm{~J}$ and $291.86 \mathrm{~J}$, and the deviation with respect to the reference motion are 12.93, 14.45 and 16.44 for cases 1,2 and 3 , respectively. It is worth of mention that the metabolical cost of transportation is higher for the case 2 than for case 3 , while the subject of case 3 has a stronger limitation of muscle capacity than the subject of case 2 . These results are 

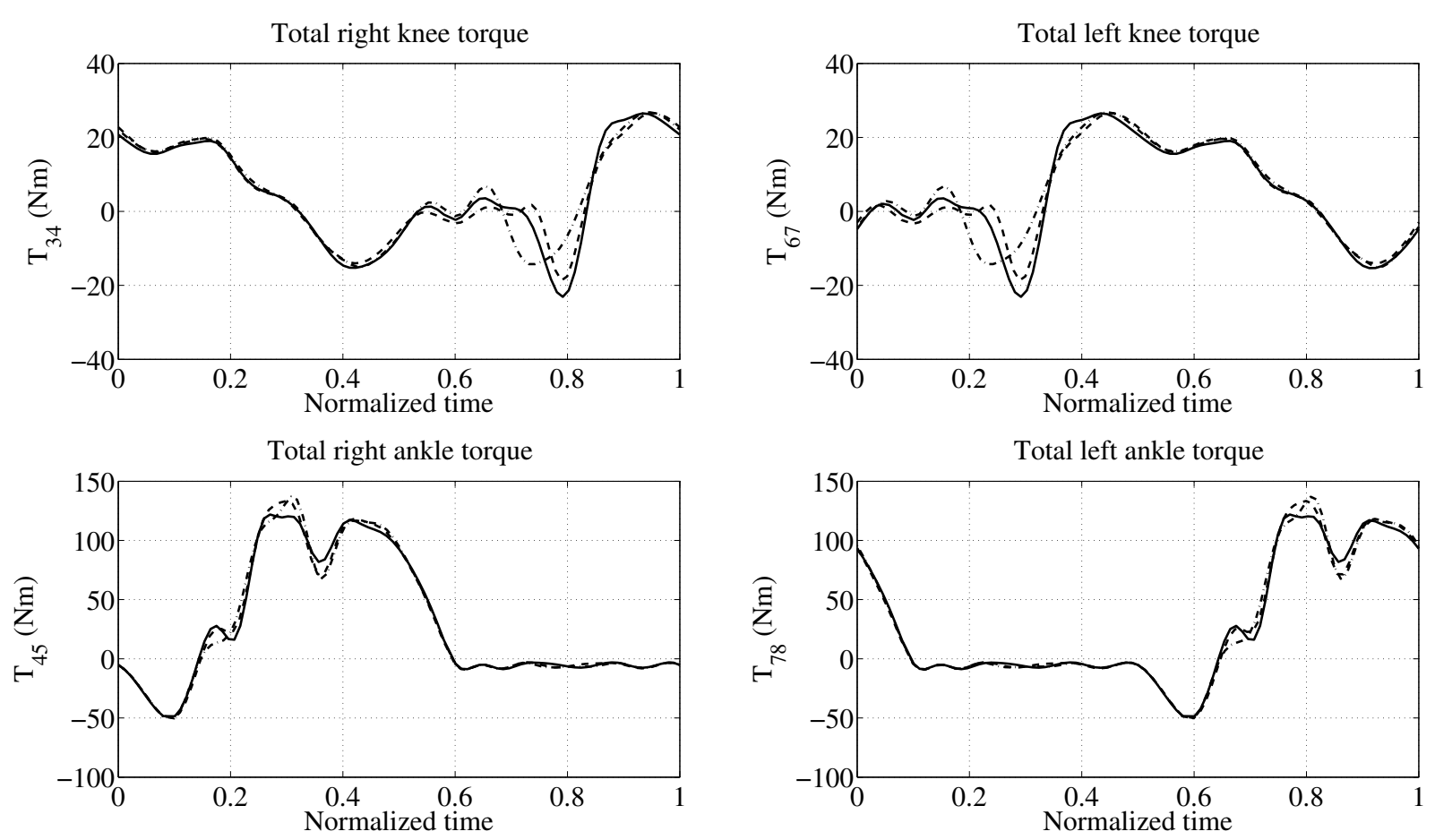

Figure 11: Total torques (orthosis joint torques plus muscle torques) obtained using cost function $f_{A}$ (solid line), cost function $f_{B}$ (dashed line) and cost function $f_{C}$ (dash-dotted line). The upper row of plots represents the net torques exerted by the orthosis and the muscles spanning the knee at the knee joints while the lower row of plots represents the net torques exerted by the orthosis flexible ankle and the muscles spanning the ankle at the ankle joints.
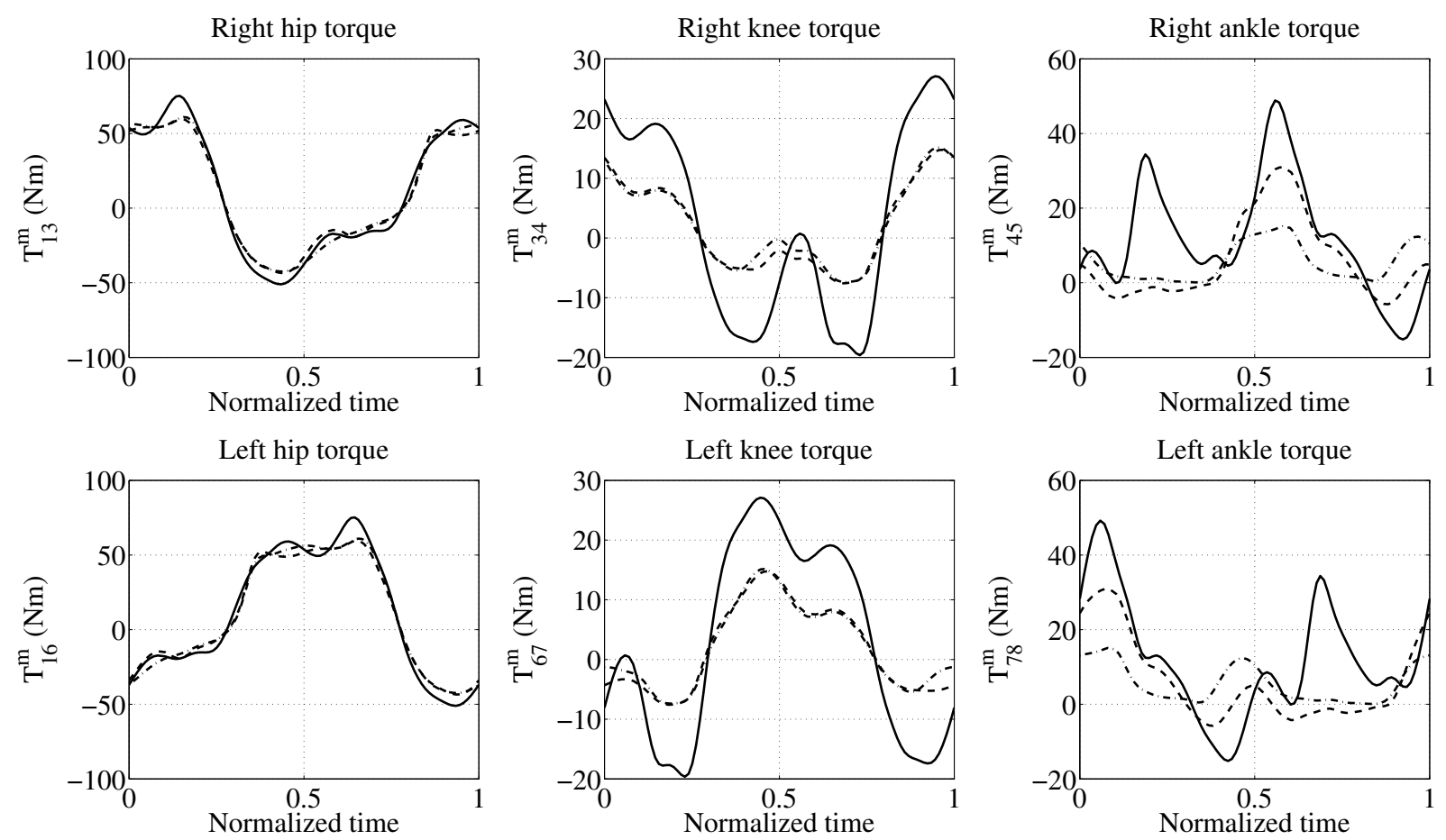

Figure 12: Muscle joint torques for case 1 (solid line), for case 2 (dashed line) and for case 3 (dash-dotted line). The upper row of plots represents the muscle joint torques in the right leg joints while the lower row of plots represents the muscle joint torques in the left leg joints. From left to right, each row shows the muscle joint torques at the hip, knee and ankle joints. 
interpreted as that the subject of case 2 experiences a higher metabolical cost than the subject of case 3 in order to have a gait pattern that is closer to the reference one than that of the subject of case 3 .

The Figure 12 shows a comparison of the net muscle torques at the joints for the different subjects simulated. As shown in the figure, the muscle torques at the hip joints are very similar for the three subjects. This may be due to the fact that the muscles actuating only at the hip joint (Iliopsoas and Gluteus) are not weakened in any of the three subjects. Regarding the knee muscle torques, a substantial difference is found between the AIS D and the AIS C subjects. Note that the knee muscle torque in the subjects with weakest muscles are obviously smaller. The same results can be seen in the ankle muscle torques. It shall be pointed out that for the weakest subject (case 3), there is still a possible muscle actuation due to Gastrocnemius muscle. In principle, the results shown in Figure 12 are reasonable since they show a muscle torque contribution that is in accordance with the level of denervation of the muscles spanning the joints.
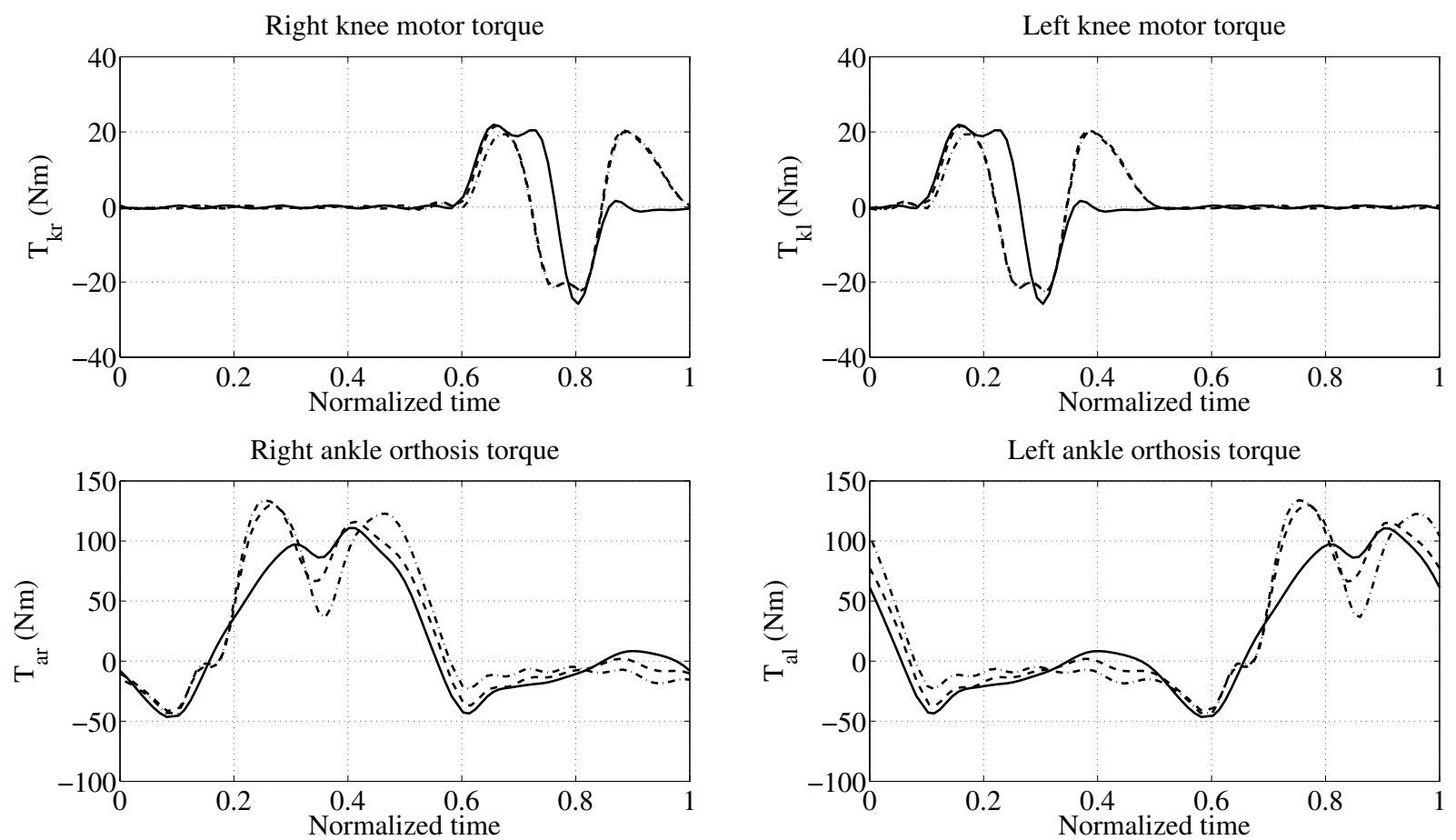

Figure 13: Orthosis joint torques for case 1 (solid line), for case 2 (dashed line) and for case 3 (dash-dotted line). The upper row of plots represents the torques exerted by the orthosis motors at the knee joints of the model while the lower row of plots represents the torques exerted by the orthosis flexible ankle at the ankle joints of the model.

Figure 13 shows the torques at the knee and ankle joints due to the two active orthoses. In accordance with the results shown in the previous figure, the motor torques at the knees are different for the AIS C and AIS D subjects, being very close one to the other those of the AIS C subjects. As shown in the upper right and left plots in the figure, the needs for motor actuation during the swing phase are different for the AIS C and AIS D subjects, being significatively large for the weakest subjects (AIS C) at the end of the swing phase. It is worth mentioning here that having the optimum motor torque histories is a very interesting issue from a design point of view. This information may help the engineer at programming the motor control of the active orthosis.

In respect of the Klenzak ankle joint (lower right and left plots in Figure 13) the torque due to the flexibility of the joint is larger for those subjects that cannot have a good muscle actuation at the ankle (cases 2 and 3). In particular, for case 3 the contribution of the Klenzak ankle joint 
needs to be larger than that of case 2 due to the incapacity of muscles Tibialis Anterior and Soleus.

A comparison of the different neural excitations obtained for the three subjects simulated is shown in Figure 14. As described before, the neural excitations remain bounded according to the weakness vectors defining cases 1,2 and 3 . It is remarkable the absence of neural excitation in Tibialis Anterior and Soleus for the subject of case 3. Interestingly, even with such a small contribution from the muscles, the gait cycle seems to be possible with the help of the active orthosis. 

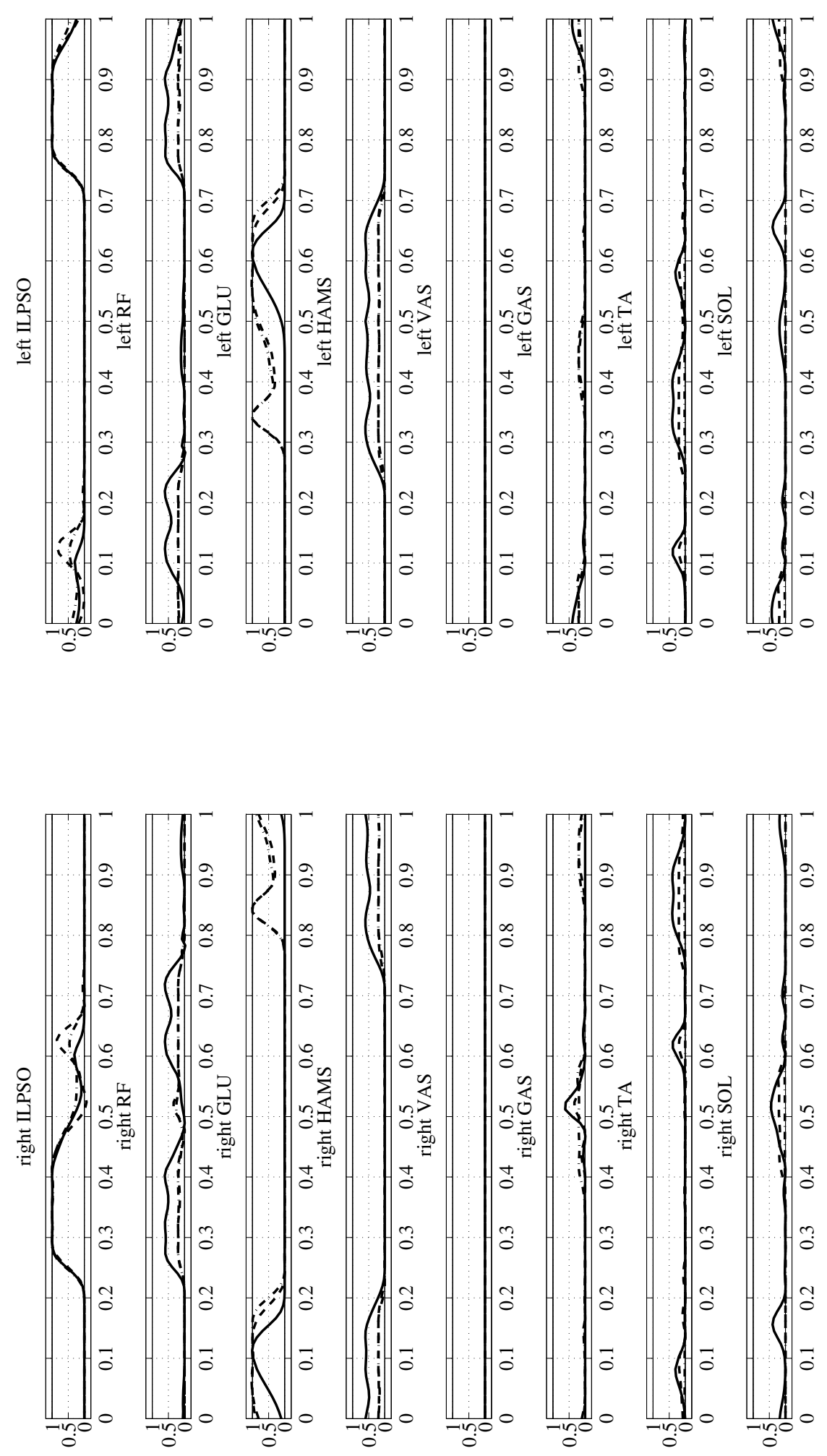

Figure 14: Neural excitations for case 1 (solid line), for case 2 (dashed line) and for case 3 (dashdotted line) for the sixteen muscles of the model depicted in Fig. 2 according to the definition by Eq. (9). The eight plots in the top part correspond to the muscles of the left leg while the eight plots on the bottom part correspond to the muscles of the right leg. 


\subsection{Stiffness requirements of the flexible ankle according to the level of injury}

This section shows how the optimization framework described before can be used to assist in the design of the active orthoses. Many computations have been carried out using different weakness vectors $\boldsymbol{p}$. This way it is possible to study the stiffness requirements at the flexible ankle joint for different levels of injury. As described in Equations (12) and (13), the flexible ankle characteristic is described in terms of two design parameters, the flexible ankle torques in neutral position, $T_{a r}^{0}$ (right) and $T_{a l}^{0}$ (left), and the flexible ankle rotational stiffness, $k_{a r}$ (right) and $k_{a l}$ (left). During the iterative solution of the optimization problem, these design parameters are allowed to vary as much as necessary to find the minimal value of the objective function. In this case, the cost function $f_{A}$ was used to search for the best set of design variables.

To represent a gradual decrease of muscle actuation capacity, the following weakness vector was defined:

$$
\boldsymbol{p}=[1, \alpha, 1, \alpha, 0.8 \cdot \alpha, 0.6 \cdot \alpha, 0.4 \cdot \alpha, 0.4 \cdot \alpha]^{T}
$$

where all the neural excitation bounds are defined in terms of one parameter, $\alpha$, that will be referred as denervation parameter hereafter. According to Equation (35), the lower the denervation parameter the weaker the subject is. Such a representation in terms of one parameter has been defined with the aim of having a representation of the optimum value a certain design variable in terms of the weakness of the subject. Of course, other weakness vector definitions could be used.
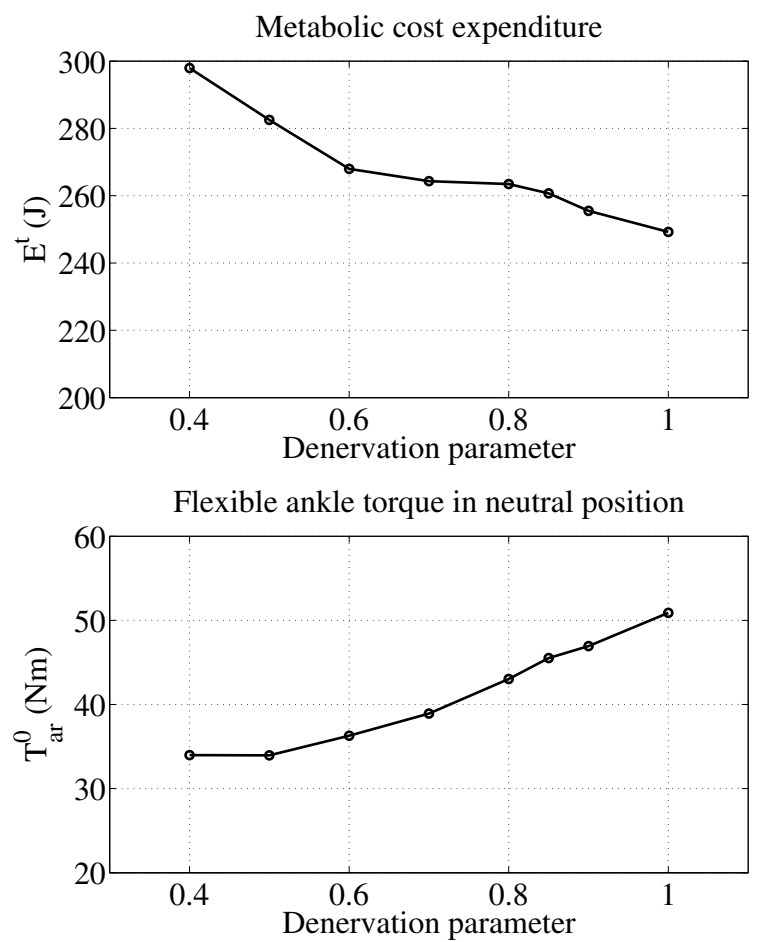
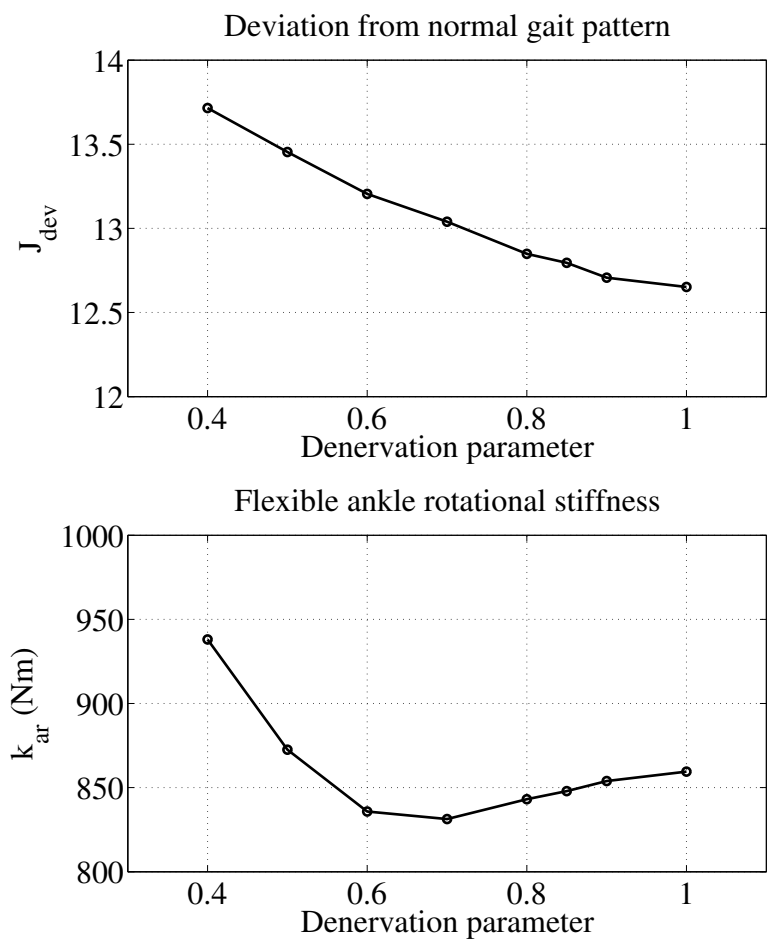

Figure 15: Metabolic expenditure (upper left plot), deviation from normal gait patterns (upper right plot), optimum ankle torque in neutral position (lower left plot) and optimum ankle rotational stiffness (lower right plot).

Figure 15 shows the relation of the optimal values obtained for the energy expenditure, the deviation from normal gait pattern, the torque in neutral position and the rotational stiffness with the denervation parameter, $\alpha$. In the upper left plot of the figure, it can be seen that the metabolic cost increases as the denervation parameter decreases, that is, as the weakness of the subject increases. While a decrease of the denervation parameter, $\alpha$, results in less neural excitation, less muscle force and, therefore, less metabolic cost, it has to be noted that the results show that $J_{d e v}$ grows as $\alpha$ decreases. It means that the subject is not able to develop a motion 
close enough to the reference motion provided, therefore performing a metabolically expensive gait cycle.

The most interesting results are shown in the lower right and left plots in Figure 15 where it is found the evolution of the optimal torque in neutral position and of the optimal rotational stiffness with the denervation parameter. These graphics show that each injured subject may have different stiffness requirements for an optimal performance of the orthosis. In particular, the optimal value of the flexible torque in neutral position decreases with the decrease in the denervation parameter while the optimal rotational stiffness shows a non-monotonic behavior. For a moderate decrease of $\alpha$ (Equation (35)) the optimal stiffness slightly decrease but, for a significant decrease in the denervation parameter $\alpha$, the optimal stiffness experience a pronounced increase.

\section{Conclusions}

This paper presents a parameter optimization method to analyse the gait of a subject with a SCI wearing two knee-ankle-foot active orthoses. In this approach, muscle forces and generalized coordinates are found by minimizing a cost function that accounts for subject's energy expenditure, and deviation from a normal gait pattern, as well as for the intensity of the orthoses actuation. In the last part of the numerical results section, two design parameters of the flexible ankle are also found by minimization of a cost function including the metabolical cost of transportation and the deviation from a normal gait pattern.

The method is mainly based on inverse dynamics, since at each iteration of the optimization algorithm an inverse dynamic problem is solved by using the motion reconstructed from the design variables. The main advantage of this approach is the complete elimination of the forward integrations of the equations of motion resulting in an efficient simulation required for design purposes.

The following list summarizes the main conclusions drawn from the analysis carried out:

- It is possible to include the subject's SCI and the orthosis main design parameters into the parameter optimization framework in order to account for different injury levels as well as to find the best set of design parameters for the orthosis.

- Three objective functions are defined based on different measures of the orthosis actuation. One of the evaluated objective functions does not take into account the orthosis actuation while the other two consider the RMS value of the mechanical power of the actuator and the RMS measure of the actuator torque. As compared to the first objective function, including the contribution of the orthosis in the objective function to be minimized results in a larger actuation of the different muscles and a less energy consumption of the actuators.

- Using a cost function considering metabolical cost of transportation and deviation from a normal gait pattern and considering different injury severities, by limiting lower limb muscle excitations, numerical results show reasonable human-orthosis motions. Moreover, the proposed optimization formulation allows to obtain human-orthosis co-actuation strategies, where different device actuation is obtained depending on how the orthosis performance is included in the cost function to be minimized.

- Simulations are also used to obtain the optimal values of two orthosis design parameters: the ankle joint stiffness and the ankle torque in neutral position, which are used to apply the dorsiflexion torque to avoid drop foot, for different levels of injury. Therefore, the results of the optimization approach allows the design of active orthoses considering energy expenditure, aesthetics of gait and orthosis mechanical performance. 
The proposed methodology opens a new perspective towards the analysis and experimental validation of human-assisted gait most helpful for the conception of advanced assistive devices for rehabilitation. Future work will be devoted to implement a more realistic foot-ground contact model, and to consider general 3D motions.

\section{Conflict of interest statement}

The authors do not have any conflicts of interest with regard to this paper and the materials contained herein.

\section{Acknowledgements}

This work was partially supported by the Spanish Ministry of Economy and Competitiveness under the project DPI2012-38331-C03-02, co-funded by the European Union through ERDF funds. The support is gratefully acknowledged.

\section{Appendix A Muscle contraction dynamics}

The force-length-velocity relation used in this work is taken from the work of Nagano and Gerritsen [25], who adopted the formulae describing the muscle contraction dynamics from van Soest and Bobbert [34] and Cole et al. [35]. For the concentric contraction phase $\left(v^{c e}<0\right)$, the CE velocity $v^{c e}$ is written as follows:

$$
v^{c e}=-\Gamma l_{o p t}^{c e}\left(\frac{\left(f_{\text {isom }}+A_{r e l}\right) B_{r e l}}{\frac{f^{c e}}{a f_{m a x}^{m}}+A_{r e l}}-B_{r e l}\right)
$$

where $\Gamma=\min (1,3.33 a)$, being $a$ the muscle activation level. To simplify the inversion of the contraction dynamics, Ackermann [6] used a constant value for factor $\Gamma$ equal to 1 obtaining consistent results. In this work, the same assumption for factor $\Gamma$ is made. In Equation (A.1), $A_{r e l}$ and $B_{r e l}$ are two muscle constant that may depend on the percentage of fast twitch fibers, see Umberger at al. [30], or training conditions, see Nagano and Gerritsen [25]. Typical values for this parameters used in [25] and [6] are $A_{r e l}=0.41$ and $B_{r e l}=5.2$. The force relative to $f_{m a x}^{m}$ produced at isometric contraction, $f_{\text {isom }}$, is

$$
f_{\text {isom }}=c\left(\frac{l^{c e}}{l_{o p t}^{c e}}\right)^{2}-2 c\left(\frac{l^{c e}}{l_{o p t}^{c e}}\right)+c+1
$$

being $c=-1 /$ width $^{2}$. Values for the width parameter can be found in Nagano and Gerritsen [25]. On another hand, in the eccentric contraction phase $\left(v^{c e}>0\right)$, the CE velocity can be written as

$$
v^{c e}=-l_{o p t}^{c e}\left(\frac{c_{1}}{\frac{f^{c e}}{a f_{\max }^{m}}+c_{2}}-c_{3}\right)
$$

being

$$
\begin{aligned}
c_{1} & =\frac{\Gamma B_{\text {rel }}\left(f_{\text {isom }}+c_{2}\right)^{2}}{\left(f_{\text {isom }}+A_{\text {rel }}\right) S_{f}} \\
c_{2} & =-f_{\text {isom }} f_{\text {asymp }} \\
c_{3} & =\frac{c_{1}}{f_{\text {isom }}+c_{2}}
\end{aligned}
$$


where $f_{\text {asymp }}$ is the asymptotic maximum force value in the eccentric phase relative to $f_{m a x}^{m}$ and $S_{f}$ is the ratio between concentric and eccentric derivatives of force with respect to $v^{c e}$. In this work $S_{f}=1$ is used in order to enforce continuity of the slope of the force-velocity curve at $v^{c e}=0$.

\section{Appendix B Tendon force-length}

The force-length curve used to model tendons in this work is as follows

$$
f^{\text {se }}\left(l^{\text {se }}\right)= \begin{cases}k^{T}\left(l^{\text {se }}-l_{\text {slack }}\right)^{2} & \text { if } \quad l^{\text {se }} \geq l_{\text {slack }} \\ 0 & \text { if } \quad l^{\text {se }}<l_{\text {slack }}\end{cases}
$$

where $k^{T}=f_{\max }^{c e} /\left(\varepsilon_{0}^{T} l_{\text {slack }}\right)^{2}$, and $l_{\text {slack }}$ is the tendon slack length for which the tendon or SE begins to resist lengthening. A typical value of $\varepsilon_{0}^{T}=4 \%$ is used in this work.

\section{References}

[1] M. Peasgood, J. McPhee, and E. Kubica. Stabilization and energy optimization of a dynamic walking gait simulation. In Proceedings of the ASME 2005 International Design Engineering Technical Conferences \& Computers and Information in Engineering, Long Beach, California, USA, September 24-28 2005.

[2] M. Ackermann and W. Schiehlen. Dynamic analysis of human gait disorder and metabolical cost estimation. Archive of Applied Mechanics, 75(10-12):569-594, 2006.

[3] R. Al Nazer, T. Rantalainen, A. Heinonen, H. Sievänen, and A. Mikkola. Flexible multibody simulation approach in the analysis of tibial strain during walking. Journal of Biomechanics, 41:1036-1043, 2008.

[4] S.E. Rodrigo, J.A.C. Ambrosio, M.P.T. Da Silva, and O.H. Penisi. Analysys of human gait based on multibody formulations and optimization tools. Mechanics Based Design of Structures and Machines, 36(4):446-477, 2008.

[5] G. Bessonnet, P. Seguin, and P. Sardain. A parametric optimization approach to walking pattern synthesis. The International Journal of Robotics Research, 24(7):523-536, 2005.

[6] M. Ackermann. Dynamics and energetics of walking with prostheses. Shaker-Verlag, Aachen, 2007.

[7] D. García-Vallejo and W. Schiehlen. 3D-Simulation of human walking by parameter optimization. Archive of Applied Mechanics, 82:533-556, 2012.

[8] B.R. Umberger. Effects of suppressing arm swing on kinematics, kinetics and energetics of human walking. Journal of Biomechanics, 41:2575-2580, 2008.

[9] S.L. Delp, F.C. Anderson, A.S. Arnold, P. Loan, A. Habib, C.T. John, E. Guendelman, and D.G. Thelen. Opensim: open-source software to create and analyze dynamic simulations of movement. IEEE Transactions on Biomedical Engineering, 54(11):1940-1950, 2007.

[10] M. Hubli and V. Dietz. The physiological basis of neurorehabilitation - locomotor training after spinal cord injury. Journal of NeuroEngineering and Rehabilitation, 10(5):1-8, 2013.

[11] A.M. Dollar and H. Herr. Lower extremity exoskeletons and active orthoses: challenges and state-of-the-art. IEEE Transactions on Robotics, 24:1-15, 2008. 
[12] J. Blaya and H. Herr. Adaptive control of a variable-impedance ankle-foot orthosis to assist drop-foot gait. IEEE Transactions on Neural Systems \& Rehabilitation Engineering, 12:24$31,2004$.

[13] T. Yakimovich, E.D. Lemaire, and J. Kofman. Engineering desing review of stance-control knee-ankle-foot orthoses. Journal of Rehabilitation Research \& Development, 46:257-267, 2009 .

[14] Font-Llagunes, J.M., Pàmies-Vilà, R., Alonso, F.J. and Lugrís, U. Simulation and design of an active orthosis for an incomplete spinal cord injured subject. Procedia IUTAM, 2:68-81, 2011.

[15] G. Sawicki and D. Ferris. A pneumatically powered knee-ankle-foot orthosis (kafo) with myoelectric activation and inhibition. Journal of NeuroEngineering and Rehabilitation, 6:623, 2009.

[16] E. Strickland. Good-bye, wheelchair. IEEE Spectrum, 49(1):30-32, 2012.

[17] A. Esquenazi, M. Talaty, A. Packel, and M. Saulino. The rewalk powered exoskeleton to restore ambulatory function to individuals with thoracic-level motor-complete spinal cord injury. American Journal of Physical Medicine \& Rehabilitacion, 91(11):911-921, 2012.

[18] Urbano Lugrís, Jairo Carlín, Alberto Luaces, and Javier Cuadrado. Gait analysis system for spinal cord-injured subjects assisted by active orthoses and crutches. Proceedings of IMechE Part K: Jorunal of Multi-body Dynamics, 227(4):363-374, 2013.

[19] T. Kurz, P. Eberhard, C. Henninger, and W. Schiehlen. From Neweul to Neweul-M ${ }^{2}$ : Symbolical equations of motion for multibody system analysis and synthesis. Multibody System Dynamics, 24(1), 2010.

[20] W. Schiehlen. Multibody system dynamics: Roots and perspectives. Multibody System Dynamics, 1:149-188, 1997.

[21] R. Riener and T. Edrich. Identification of passive elastic joint moments in the lower extremities. Journal of Biomechanics, 32:539-544, 1999.

[22] R.B. Stein, E.P. Zehr, M.K. Lebiedowska, D.B. Popovic, A. Scheiner, and H.J. Chizeck. Estimating mechanical parameters of leg segments in individuals with and without physical disabilities. IEEE Transactions on Rehabilitation Engineering, 4(3):201-211, 1996.

[23] A. Hill. The heat of shortening and the dynamic constants of muscle. Proceedings of the Royal Society of London B, 126:136-195, 1938.

[24] F. Zajac. Muscle and tendon: Properties, models, scaling and applications to biomechanics and motor control. Critical Reviews in Biomedical Engineering, 17:359-411, 1989.

[25] A. Nagano and K.G.M. Gerritsen. Effects of neuromuscular strength training on vertical jumping performance - a computer simulation study. Journal of Applied Biomechanics, $17: 113-128,2001$.

[26] J. M. Winters. Hill-based muscle models: a system engineering perpective. In J. M.Winters and S. L.-Y.Woo, editors, Multiple Muscle Systems, pages 69-93. Springer-Verlag, 1990.

[27] Alonso, F.J., Romero, F., Pàmies-Vilà, R., Lugrís, U., and Font-Llagunes, J.M. A simple approach to estimate muscle forces and orthosis actuation in powered assisted walking of spinal cord-injured subjects. Multibody System Dynamics, 28(1-2):109-124, 2012. 
[28] J.G. Hincapie, D. Blana, E.K. Chadwick, and R.F. Kirsch. Musculoskeletal Model-Guided, Customizable Selection of Shoulder and Elbow Muscles for a C5 SCI Neuroprosthesis. IEEE Transactions on Neural Systems and Rehabilitation Engineering, 16(3):255-263, 2008.

[29] S.J. Sober, J.M. Stark, D.S. Yamasaki, and W.W. Lytton. Receptive field changes after strokelike cortical ablation: A role for activation dynamics. Journal of Neurophysiology, 78(6):3438-3443, 1997.

[30] B.R. Umberger, K.G.M. Gerritsen, and P.E. Martin. A model of human muscle energy expendidure. Computer Methods in Biomechanics and Biomedical Engineering, 6(2):99-111, 2003.

[31] R.A. Brand, D.R. Pedersen, and J.A. Friederich. The sensitivity of muscle force predictions to changes in physiologic cross-sectional area. Journal of Biomechanics, 19(8):589-596, 1986.

[32] M. Ackermann and H. Gros. Measurements of human gaits. Technical Report Zwischenbericht ZB-144, Institute of Engineering and Computational Mechanics, University of Stuttgart, 70550 Stuttgart, 2005.

[33] G. Strang. Linear algebra and its applcations, $2^{\text {nd }}$ Edition. Academic Press, New York, 1980 .

[34] A.J. van Soest and M.F. Bobbert. The contribution of muscle properties in the control of explosive movements. Biological Cybernetics, 69:195-204, 1993.

[35] G.K. Cole, A.J. van den Bogert, W. Herzog, and K.G.M. Gerritsen. Modeling of force production in skeletal muscle undergoing stretch. Journal of Biomechanics, 29:1091-1104, 1996. 